\title{
Theory of flavors: String compactification
}

\author{
Jihn E. Kim \\ Department of Physics, Kyung Hee University, 26 Gyungheedaero, \\ Dongdaemun-Gu, Seoul 02447, Republic of Korea, \\ and Center for Axion and Precision Physics Research (Institute of Basic Science), KAIST Munji Campus, \\ 193 Munjiro, Daejeon 34051, Republic of Korea, and Department of Physics and Astronomy, \\ Seoul National University, 1 Gwanakro, Gwanak-Gu, Seoul 08826, Republic of Korea
}

(Received 4 June 2018; published 5 September 2018)

\begin{abstract}
We present a calculating method for the quark and lepton mixing angles. After a general discussion in field theoretic models, we present a working model from a string compactification through $\mathbf{Z}_{12-I}$ orbifold compactification. It is beyond presenting just three families of the standard model but is the first example from string compactification successfully fitting to the observed data. Assuming that all Yukawa couplings from string compactification are real, we also comment on a relation between the $C P$ phases in the Jarlskog determinants obtained from the Cabibbo-Kobayashi-Maskawa and Pontecorvo-MakiNakagawa-Sakada matrices. The flipped SU(5) model leads to the doublet-triplet splitting and possible proton decay operators. It is shown that the vacuum expectation values can be tuned such that the proton lifetime is long enough.
\end{abstract}

DOI: $10.1103 /$ PhysRevD.98.055005

\section{INTRODUCTION}

"How is the current allocation of flavors realized?" is the most urgent and also interesting theoretical problem in the standard model (SM). Extension of the SM to grand unification (GUT) and string models [1,2] continues to require to solve this flavor problem. Gauge symmetries as family groups should satisfy the anomaly freedom, which can be achieved in extended GUTs [3] and in models without anomaly [4]. Not to worry about the gauge anomalies, sometimes global symmetries are used for the family groups [5-7]. It has been reviewed at several places $[8,9]$.

In the SM, the difference of families is manifested in the Cabibbo-Kobayashi-Maskawa (CKM) matrix in the quark sector $[10,11]$ and in the Pontecorvo-Maki-NakagawaSakada (PMNS) matrix in the leptonic sector $[12,13]$. To relate the left $(\mathrm{L})$ and $\operatorname{right}(\mathrm{R})$ mixing angle parameters, the flavor group $G_{f}$ has been introduced to obtain more relations between flavor parameters [14-17]. In most cases, a factor flavor group $G_{f}$ is introduced in addition to the SM or GUT. On the other hand, an attractive mechanism is to unify all the fermion representations in an irreducible set of $\mathrm{SU}(N)$ representations of an extended GUT [3,18-20].

Published by the American Physical Society under the terms of the Creative Commons Attribution 4.0 International license. Further distribution of this work must maintain attribution to the author(s) and the published article's title, journal citation, and DOI. Funded by SCOAP ${ }^{3}$.
The $E_{8} \times E_{8}^{\prime}$ gauge group can be considered to belong to this class but in ten dimensions. Compactification of six extra dimensions may be the key to the unification of families in ten dimensional superstring models.

A notable difference between the CKM and the PMNS matrices lies in the fact that in the CKM matrix the large elements are located in the diagonal entries while it is not so in the PMNS matrix. So, for the CKM parameters the quark mass ratios were used before $[14,15]$. On the other hand, for the PMNS parameters non-Abelian discrete groups are used $[21,22]$. One may say that there is one similarity in the $C P$ phases of the CKM and PMNS matrices. The CKM phase is close to 90 degrees in the Kim-Seo (KS) parametrization [23] and the PMNS phase is -90 degrees (but with a large error bars) [24]. Even, there exists an attempt to unify these $C P$ phases [25].

To reduce the number of parameters in the flavor sector, family symmetries can be used. Simple ones are $\mathrm{U}(1)$ groups. But, to introduce a hierarchy, vacuum expectation values (VEVs) of the SM singlets are suggested, which is known as the Froggatt-Nielsen (FN) mechanism [26].

In this paper, we study singlet representations beyond the SM based on family symmetry groups. For various reasons in field theoretic models, we consider $\mathrm{U}(1)^{2}$ among which one is anomalous and the other is anomaly free. We attempt to obtain singlets from the orbifold compactification of the $E_{8} \times E_{8}^{\prime}$ heterotic string [27] based on the simplest $\mathbf{Z}_{12-I}$ lattice [28,29]. Fixed points of 13 prime orbifolds listed in [30] shows that the $\mathbf{Z}_{12-I}$ lattice can be 
considered to be the simplest because there are only three fixed points. ${ }^{1}$

Yet the clearest statement to date is that standardlike models are exceedingly rare [31,32]. The degree of acceptable standard-like models can be guessed from minilandscape studies of $\mathbf{Z}_{6-I I}$ models [33,34]. In [34], it was shown that acceptable model is $\mathrm{O}(1)$ out of $\mathrm{O}\left(10^{6}\right)$. Even in this case, one has to check all elements of the CKM and PMNS matrices.

In Sec. II, we briefly recapitulate the fermion mass structure: Dirac fermions of charged leptons and quarks, and Majorana fermions for the SM neutrinos. We set up the scheme to use Weyl fermions to express both the Dirac and Majorana masses pressented in subsequent sections. Those who are familiar to these can skip this section. In Sec. III, we present a beyond-SM with two U(1)'s toward useful fermion mass textures, where one is $\mathrm{U}(1)_{\text {anom }}$ global symmetry for the "invisible" axion and the other is anomaly free gauged U(1). In Sec. IV, we obtain a successful flavor structure from $\mathbf{Z}_{12-I}$ orbifold compactification. The 3rd family is assumed to be the one from the untwisted sector $U$. Section $\mathrm{V}$ is a conclusion.

\section{FERMION MASSES}

For continuous parameters of transformation, let us begin with the axial-vector currents of fermions

$$
J_{\Gamma}^{\mu}=\bar{\Psi} \gamma^{\mu} \gamma_{5} \Gamma \Psi
$$

where $\Gamma$ is a charge operator and $\Psi$ is a column vector of three fermions (families). The divergence of the current is

$$
\partial_{\mu} J_{\Gamma}^{\mu}=\frac{A_{I}}{32 \pi^{2}} G^{I} \tilde{G}^{I}+2 \mu J_{\Gamma}^{5}
$$

where $A_{I}$ is the anomaly coefficient of the gauge fields $A_{\mu}^{I}$ for the gauge group $\mathrm{SU}(N)_{I}$, and $J_{\Gamma}^{5}$ depends on the masses of fermions,

$$
J_{\Gamma}^{5}=\frac{1}{\mu} \bar{\Psi} i \gamma_{5} M_{\Gamma} \Psi
$$

where $\mu$ is a mass scale and $M$ is the mass matrix in the flavor basis. The anomaly term is a flavor singlet which can be written in terms of a flavor singlet quark fields in the $\theta$-vacuum, e.g., for two flavors in $\mathrm{SU}(3)_{c}$ [35],

\footnotetext{
${ }^{1}$ The $\mathbf{Z}_{3}$ orbifold, seemingly looking very simple, has 27 fixed points. Being simple, $\mathbf{Z}_{12-I}$ may not be general enough, but can present the basic working principles in terms of small number of fields.
}

$G^{I} \tilde{G}^{I} \propto \sqrt{\frac{m_{u} m_{d}}{2 \cos \theta+\left(1+Z^{2}\right) / Z}}\left(\bar{u} i \gamma_{5} u+\bar{d} i \gamma_{5} d\right) \sin \theta$

where $Z=m_{u} / m_{d} \simeq \frac{1}{2}$. Obviously, the anomalous and anomaly free terms give nonzero trace for the fermion mass matrix. In Ref. [9], two U(1) symmetries were considered, one anomalous and the other anomaly-free. The anomalous global symmetry is to introduce the socalled "invisible" axion. Since the sum of quark masses is nonzero and large $\mathrm{O}\left(m_{t}\right)$, we also attempt to have the anomalous U(1). The anomalous U(1) must be a global symmetry and the anomaly free part can be a gauge symmetry. Let us start using two component fermions to write down mass terms.

\section{A. Weyl fermions}

A four component Dirac spinor, e.g., for the electron field, can be split into two Weyl spinors $\xi$ and $\eta$,

$$
\psi_{e}=\left(\begin{array}{c}
e_{L} \\
e_{R}
\end{array}\right)=\left(\begin{array}{c}
e_{L 1} \\
e_{L 2} \\
e_{R 1} \\
e_{R 2}
\end{array}\right) \rightarrow\left(\begin{array}{c}
\xi_{L} \\
\eta_{R}
\end{array}\right) .
$$

Gauge interactions do not change the chirality. Quantum fild $e_{L}$ destroys a L-handed electron and creates a R-handed positron. But, $e_{L}$ has nothing to do with destroying a R-handed electron $e_{R}$ and creating a L-handed positron. On the other hand, the antiparticle of the L-handed electron $e_{L}=\left(e_{L 1}, e_{L 2}, 0,0\right)^{T}$ is

$$
\begin{aligned}
\left(e_{L}\right)^{c} & =\left(\begin{array}{c}
e_{L 1} \\
e_{L 2} \\
0 \\
0
\end{array}\right)^{c}=\left(\frac{1+\gamma_{5}}{2} e\right)^{c}=i \sigma_{2}\left(\frac{1+\gamma_{5}^{*}}{2} e^{*}\right) \\
& =\frac{1-\gamma_{5}}{2}\left(\begin{array}{c}
0 \\
0 \\
e_{L 2}^{*} \\
-e_{L 1}^{*}
\end{array}\right)
\end{aligned}
$$

which is a R-handed field. This R-handed field destroys $\mathrm{R}$-handed positron and creates L-handed electron. With these two Weyl fields, we can destroy L- and R-electrons and create L- and R-positrons, which is done by a fourcomponent Direc electron. Thus, two Weyl fields are enough at this stage. With the Weyl field $\xi$, let us construct a Lorentz invariant $\epsilon_{i j} e_{L i} e_{L j}$. It is the mass term but the electron number is broken by this term. So, for charged particles, one Weyl field cannot be massive. For neutrinos, one Weyl field can give a mass term which is known to be 
Majorana mass. In this paper, we will use Weyl fields even for expressing Majorana masses,

$$
\epsilon_{i j}\left(\xi^{T}\right)^{i} \xi^{j}=\xi^{T}\left(\begin{array}{cc}
0 & 1 \\
-1 & 0
\end{array}\right) \xi=\xi^{T} i \sigma_{2} \xi=\xi^{T} \gamma_{1} \gamma_{3} \xi .
$$

For a Dirac mass we use the opposite chirality, i.e.,

$$
\xi_{L}^{T i}=\epsilon^{i k} \bar{\eta}_{k R} \rightarrow \epsilon_{i j} \epsilon^{j k} \bar{\eta}_{k R}=\bar{\eta}_{i R}
$$

so that (7) becomes

$$
\bar{\eta}_{R} \xi_{L}
$$

Assigning the same charge conjugation for $\xi$ fields in (7), the Majorana mass term breaks $C$, but the Dirac mass term (9) can preserve $C$ by assigning the same $C$ 's for $\xi$ and $\eta$. Discussing both Majorana and Dirac masses, using the Weyl fermion is therefore simple enough.

\section{B. $m_{b} \simeq m_{\tau}$ and Georgi-Jarlskog relation}

The observed ratio of the third family masses $m_{b} / m_{\tau} \simeq$ $4.5 / 1.5 \approx 3$ hints that $m_{b} \simeq m_{\tau}$ at the unification scale. The factor 3 arises by renormalization group evolution [36]. In the Georgi-Glashow (GG) SU(5) [37], $\overline{\mathbf{1 0}}_{f} \mathbf{5}_{f} \mathbf{5}_{H}$ gives the same mass to $b$ and $\tau$ by $\left\langle\mathbf{5}_{H}\right\rangle$ and it is considered to be a success of the GUT [38]. For the muon and strange quark, however, there is a big problem in the GG SU(5) model. The low energy mass ratio at $100 \mathrm{MeV}$ is $m_{s} / m_{\mu} \approx 1$ while the renormalization group evolution expects it to be 3 if $m_{s} \simeq m_{\mu}$ at the unification scale. If $m_{s} / m_{\mu} \simeq \frac{1}{3}$ at the unification scale, then the low energy mass ratio is understandable. But, this is a big problem with Higgs quintets only. One way out is the Georgi-Jarlskog relation introducing a big Higgs representation $\mathbf{4 5}_{H}$ [39]. If $\left\langle\mathbf{4 5}_{H}\right\rangle$ is the leading contribution to the second family fermions in the GG model, then $m_{s} / m_{\mu} \simeq \frac{1}{3}$ is obtained at the unification scale. To present a rationale for $\mathbf{4 5}_{H}$ for the needed mass matrix texture, two U(1)'s were suggested long time ago [9].

\section{Flipped SU(5)}

Our terminology of flipped $\mathrm{SU}(5)$ is a rank 5 gauge group $\mathrm{SU}(5)_{\text {flip }}=\mathrm{SU}(5) \times \mathrm{U}(1)_{X}$. Representations will be denoted as $\mathrm{SU}(5)_{\mathrm{U}(1)}$. In the flipped $\mathrm{SU}(5)$ [40,41], masses of charged leptons and $d$-type quarks are not related, which is considered to be a merit in relating masses. In string compactification, reasonable supersymmetric SM's are obtained from compactification of heterotic string. The reason is the following. For $N=1$ supersymmetric (SUSY) massless fields, only the completely antisymmetric representations are allowed with one compactification scale from heterotic string [28]. If the Higgs fields breaking a GUT group appear as massless spectra, then there is no adjoint representation at the GUT scale which is needed for breaking the GG SU(5) or $\mathrm{SO}(10)$ GUT $[42,43]$ or some Pati-Salam (PS) [44] gauge groups. ${ }^{2}$ In SU(5) flip, the representation $\mathbf{1 0}_{+1} \oplus \overline{\mathbf{1 0}}_{-1}$ can break the rank-5 SU(5) flip down to the rank-4 SM gauge group. At the GUT level, therefore only the flipped SU(5) is actually realized in several string compactifications [1,29,46,47].

\section{U(1) $)_{\text {anom }} \times \mathrm{U}(1)_{\text {fr }}$ FAMILY SYMMETRY}

We introduce supersymmetry and two $\mathrm{U}(1)$ gauge symmetries, $\mathrm{U}(1)_{\text {anom }} \times \mathrm{U}(1)_{\text {fr }}$, where $\mathrm{U}(1)_{\text {anom }}$ is anomalous and $\mathrm{U}(1)_{\mathrm{fr}}$ is free of gauge anomalies. Dangerous dimension-4 superpotential of the 1st family members triggering proton decay is

$$
q_{1} q_{1} q_{1} l_{1}
$$

where the subscript 1 denotes the first family. $\mathrm{U}(1)_{B-L}$ allows the above superpotential but $\mathrm{U}(1)_{\text {anom }}$ or $\mathrm{U}(1)_{\text {fr }}$ may not allow it. Thus, the extra U(1)'s may be useful forbidding some unwanted proton decay operators. In string compactification, one has to check the $\mathrm{U}(1)_{\text {anom }} \times \mathrm{U}(1)_{\text {fr }}$ quantum numbers of the first family members to see if the unwanted proton decay operators are forbidden. If the proton decay problem is safe, one can consider the superpotentials generating fermion masses.

The mass eigenstates of quarks, $q^{m}$, are related to the weak eigenstates by L- and R-unitary matrices, $U$ and $V$,

$$
\begin{aligned}
& q_{d, u L}^{m}=U_{d, u} q_{d, u L}^{w} \\
& q_{d, u R}^{m}=V_{d, u} q_{d, u R}^{w},
\end{aligned}
$$

and the charged $W_{\mu}^{+}$coupling for the L-handed quark doublets is

$$
W=U_{u}^{\dagger} U_{d},
$$

which is the CKM matrix.

\section{A. Effects of $U(1)$ anom on the texture of mass matrix}

To see the essence, let us consider two families of quarks. Let us choose the basis where $Q_{\mathrm{em}}=+\frac{2}{3}$ quarks are already mass eigenstates. Then, the mass matrices of weak and mass eigenstates of $Q_{\mathrm{em}}=-\frac{1}{3}$ quarks are related by $M_{d}^{w}=V_{d}^{\dagger} M_{d}^{m} U_{d}$. Parametrizing the unitary matrices as

\footnotetext{
${ }^{2}$ The electroweak PS gauge group $\mathrm{SU}(2)_{L} \times \mathrm{SU}(2)_{R} \times$ $\mathrm{U}(1)_{B-L}$ is broken by a GUT scale VEV to $\mathrm{SM} \times \mathrm{U}(1)_{B-L}$, needing an adjoint representation not to reduce the rank. Usually, it is denoted as $\Delta=(\mathbf{1}, \mathbf{3}, 0)$. A VEV of an adjoint representation does not reduce the rank of the gauge group. But, note that an adjoint representation is possible in some scenarios in $\mathbf{Z}_{6-I I}$ by introducing two compactification scales for $N=2$ SUSY in an interim effective 5 dimensions [45].
} 


$$
U_{d}=\left(\begin{array}{cc}
c_{1} & s_{1} \\
-s_{1} & c_{1}
\end{array}\right), \quad V_{d}=\left(\begin{array}{cc}
c_{2} & s_{2} \\
-s_{2} & c_{2}
\end{array}\right)
$$

where $c_{i}=\cos \theta_{i}$ and $s_{i}=\sin \theta_{i}$ for $i=1,2$. Thus, $M_{d}^{w}$ is given by

$M_{d}^{w}=\left(\begin{array}{ll}c_{1} c_{2} m_{d}+s_{1} s_{2} m_{s}, & s_{1} c_{2} m_{d}-c_{1} s_{2} m_{s} \\ c_{1} s_{2} m_{d}-s_{1} c_{2} m_{s}, & s_{1} s_{2} m_{d}+c_{1} c_{2} m_{s}\end{array}\right)$,

where $m_{d}$ and $m_{s}$ are eigenvalues of the mass matrix. If any one element of Eq. (14) is zero, then $\theta_{1}$ and $\theta_{2}$ are related. Weinberg's choice [14] is $m_{d} \rightarrow-m_{d}$ and $\left(M_{d}^{w}\right)_{11}=0$, leading to $s_{1} s_{2} / c_{1} c_{2}=m_{d} / m_{s}$. Since $\sin \theta_{C} \simeq \sqrt{m_{d} / m_{s}}$ numerically, we use the freedom in $V$ and choose $s_{2} / c_{2}=s_{1} / c_{1}$, which means that the R-handed fields transform in the same way as the L-handed fields. This implies that under any extra $\mathrm{U}(1)$ gauge group the gauge transformations of the L- and R-handed fields are identical. Thus, extra U(1)'s should be free of gauge anomalies. Therefore, if we do not consider extra quarks beyond the SM the Fritzsch texture [15], following Ref. [14], is not valid with the U(1) anom gauge group. So, it is appropriate to introduce heavy quarks to have $\mathrm{U}(1)_{\text {anom }}$ together with the Fritzsch texture.

Presence of $\mathrm{U}(1)_{\text {anom }}$ gauge group requires a difference between $U_{d}$ and $V_{d}$. To reduce one more parameter, Wilczek and Zee [16] choose $\left(M_{d}^{w}\right)_{12}=0$ with $s_{2} / c_{2} \simeq\left(s_{1} / c_{1}\right)^{3}$, which is consistent with the presence of $\mathrm{U}(1)_{\text {anom }}$ gauge group. Namely, in the presence of $\mathrm{U}(1)_{\text {anom }}$ gauge group, we must choose $V$ differently from $U$ even for three families.

Similarly, let us consider two families of leptons where charged lepton mass matrix is already diagonalized. Then, the mass matrices of weak and mass eigenstates of Majorana neutrinos are related by $M_{\nu}^{w}=U_{\nu}^{T} M_{\nu}^{m} U_{\nu}$. Thus, $M_{\nu}^{w}$ is given by

$M_{\nu}^{w}=\left(\begin{array}{cc}C_{1}^{2} m_{\nu_{e}}+S_{1}^{2} m_{\nu_{\mu}}, & -C_{1} S_{1}\left(m_{\nu_{\mu}}-m_{\nu_{e}}\right) \\ -C_{1} S_{1}\left(m_{\nu_{\mu}}-m_{\nu_{e}}\right), & S_{1}^{2} m_{\nu_{e}}+C_{1}^{2} m_{\nu_{\mu}}\end{array}\right)$,

where $C_{1}=\cos \Theta_{1}$ and $S_{1}=\sin \Theta_{1}$. Since the mixing angle of the second and the third neutrinos is large, we can approximate $C_{1} \simeq S_{1}=1 / \sqrt{2}$. In this case, the mass matrix is of the form ${ }^{3}$

$$
M_{\nu}^{w}=\left(\begin{array}{cc}
A, & -B \\
-B, & A
\end{array}\right)
$$

\footnotetext{
${ }^{3}$ This case with two parameters is including the possibility of family indices carried by Higgs fields. If family indices of Higgs fields are independent from the family index of quark and leptons, then there must be one parameter.
}

TABLE I. Charges of up type quarks.

\begin{tabular}{cccccccr}
\hline \hline & $\bar{u}_{1 R}$ & $\bar{u}_{2 R}$ & $\bar{u}_{3 R}$ & $u_{1 L}$ & $u_{2 L}$ & $u_{3 L}$ & $\sigma$ \\
\hline$Q_{\text {anom }}$ & +2 & +1 & 0 & +4 & +2 & 0 & -3 \\
\hline \hline
\end{tabular}

where $A=\left(m_{\nu_{\mu}}+m_{\nu_{e}}\right) / 2$ and $B=\left(m_{\nu_{\mu}}-m_{\nu_{e}}\right) / 2$, which has the permutation symmetry $S_{2}$ between the second and the third family indices. The useful discrete symmetries of $[21,22]$ contain this $S_{2}$ as a subgroup. In this case of introducing $\mathrm{U}(1)_{\text {anom }}$, where we introduced only L-handed neutrinos, the anomaly freedom must be satisfied by the quantum numbers of the first family leptons or by heavy leptons.

\section{B. Quark mass matrices}

Let us begin with the diagonalized Dirac masses of the form (9) for $Q_{\mathrm{em}}=+\frac{2}{3}$ quarks,

$$
\begin{aligned}
& \xi_{1 L} \quad \xi_{2 L} \quad \xi_{3 L} \\
& M_{u}^{\mathrm{diag}}=\begin{array}{l}
\bar{\eta}_{1 R} \\
\bar{\eta}_{2 R} \\
\bar{\eta}_{3 R}
\end{array}\left(\begin{array}{ccc}
m_{u} & 0 & 0 \\
0 & m_{c} & 0 \\
0 & 0 & m_{t}
\end{array}\right) \text {, }
\end{aligned}
$$

where $\bar{\eta}_{R}=\bar{q}_{u R}^{m}$ and $\xi_{L}=q_{u L}^{m}$. The diagonal form (17) with the needed hierarchy can be obtained by the U(1) charges of Table I,

$$
M_{u} \propto\left(\begin{array}{ccc}
\sigma^{2} & 0 & 0 \\
0 & \sigma & 0 \\
0 & 0 & 1
\end{array}\right)
$$

where $\propto \sigma$ is a SM singlet field carrying $Q=-3$. The mass term for up-type quarks is

$$
\bar{q}_{u R}^{m} M_{u}^{\mathrm{diag}} q_{u L}^{m}=\bar{q}_{u R}^{w} V_{u}^{\dagger} M_{u}^{\mathrm{diag}} U_{u} q_{u L}^{w} .
$$

Of course, $V_{u}=U_{u}=\mathbf{1}$.

The mass matrix for $Q_{\mathrm{em}}=-\frac{1}{3}$ quarks is

$$
\bar{q}_{d R}^{m} M_{d}^{\mathrm{diag}} q_{d L}^{m}=\bar{q}_{d R}^{w} V_{d}^{\dagger} M_{d}^{\mathrm{diag}} U_{d} q_{d L}^{w},
$$

with

$$
M_{d}=V_{d}^{\dagger}\left(\begin{array}{ccc}
m_{d} & 0 & 0 \\
0 & m_{s} & 0 \\
0 & 0 & m_{b}
\end{array}\right) U_{d}
$$


In this paper, we use the KS parametrization [23] of the CKM matrix $W_{\mathrm{KS}}$ where $\delta_{\mathrm{CKM}}=\frac{\pi}{2}$ is simple, ${ }^{4}$

$$
W_{\mathrm{KS}}=U_{d}=\left(\begin{array}{ccc}
c_{1}, & +s_{1} c_{3}, & +s_{1} s_{3} \\
-c_{2} s_{1}, & +c_{1} c_{2} c_{3}+s_{2} s_{3} e^{-i \delta}, & +c_{1} c_{2} s_{3}-s_{2} c_{3} e^{-i \delta} \\
-s_{1} s_{2} e^{i \delta}, & -c_{2} s_{3}+c_{1} s_{2} c_{3} e^{i \delta}, & +c_{2} c_{3}+c_{1} s_{2} s_{3} e^{i \delta}
\end{array}\right),
$$

with the unitary matrix for R-handed fields in the diagonalization process parametrized by another 4 parameters

$$
V_{d}^{\dagger}=\left(\begin{array}{ccc}
c_{4}, & -c_{5} s_{4}, & -s_{4} s_{5} e^{-i \Delta} \\
+s_{4} c_{6}, & +c_{4} c_{5} c_{6}+s_{5} s_{6} e^{i \Delta}, & -c_{5} s_{6}+c_{4} s_{5} c_{6} e^{-i \Delta} \\
+s_{4} s_{6}, & +c_{4} c_{5} s_{6}-s_{5} c_{6} e^{i \Delta}, & +c_{5} c_{6}+c_{4} s_{5} s_{6} e^{-i \Delta}
\end{array}\right) .
$$

Then, we obtain $V_{d}^{\dagger} M_{d}^{\text {diag }} U_{d}$ as

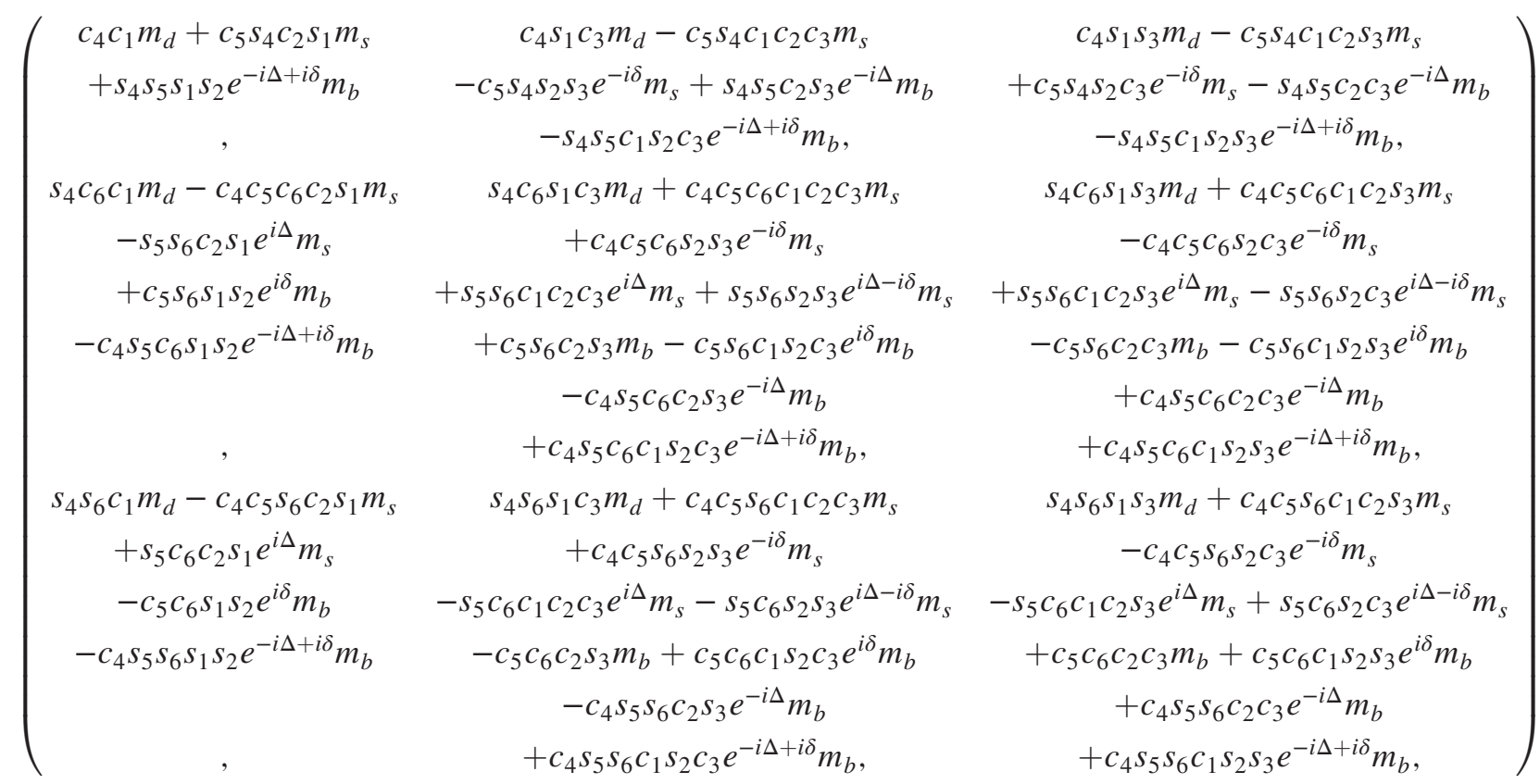

Change the sign $m_{d} \rightarrow-m_{d}$, and to reduce the number of parameters let us choose parameters of R-fields as

$$
\frac{s_{4}}{c_{4}}=\frac{s_{1}}{c_{1}}, \quad \frac{s_{5}}{c_{5}}=\frac{m_{s}}{m_{b}} \frac{s_{2}}{c_{2}}, \quad s_{6}=0, \quad \Delta=\delta .
$$

Note that $s_{4} \gg s_{1} s_{2}^{2}$. Then, keeping the largest terms in the weak basis mass matrix,

$$
\begin{aligned}
M_{d}^{w} \simeq\left(\begin{array}{ccc}
0 & -s_{1} c_{5} c_{1} c_{2}^{-1} c_{3} m_{s}, & -c_{5} c_{1} s_{1} c_{2}^{-1} s_{3} m_{s}, \\
-c_{1} c_{5} s_{1} c_{2}^{-1} m_{s}, & +c_{1} c_{5} c_{1} c_{3} c_{2}^{-1} m_{s}, & +c_{4} c_{5} c_{1} s_{3} c_{2}^{-1} m_{s}, \\
-c_{5} s_{1} s_{2} e^{i \delta} m_{b}, & -c_{5} c_{2} s_{3} m_{b}+c_{5} c_{1} s_{2} c_{3} e^{i \delta} m_{b}, & +c_{5} c_{2} c_{3} m_{b},
\end{array}\right) \\
\simeq \frac{1}{c_{5} c_{2} c_{3} m_{b}}\left(\begin{array}{ccc}
0, & -4.43 \times 10^{-3}, & -0.690 \times 10^{-4} \\
-4.43 \times 10^{-3}, & 1.918 \times 10^{-2}, & 2.99 \times 10^{-4} \\
-0.9008 \times 10^{-2} e^{i \delta}, & -1.557 \times 10^{-2}+3.90 \times 10^{-2} e^{i \delta}, & 1
\end{array}\right)
\end{aligned}
$$

\footnotetext{
${ }^{4}$ As stressed in [23], the $C P$ phase in the CKM matrix is close to 90 degrees if we parametrize it by $W_{\mathrm{Ks}}$. The phase $\delta$ is the phase in the Jarlskog determinant [48].
} 
where we used

$$
\begin{aligned}
& m_{s}=93.8 \mathrm{MeV}, \quad m_{b}=4.65 \mathrm{GeV}, \quad \frac{m_{s}}{m_{b}}=0.0202, \\
& s_{1}=0.2252, \quad c_{1}=0.9743, \quad s_{2}=0.0400, \quad c_{2}=0.9992, \quad s_{3}=0.01557, \quad c_{3}=0.9999, \\
& \frac{s_{5}}{c_{5}}=0.809 \times 10^{-5}, \quad \frac{s_{4}}{c_{4}}=\frac{s_{1}}{c_{1}} .
\end{aligned}
$$

In Eq. (26), the (32) element can be $4.2 \times 10^{-2} e^{i \delta^{\prime}}$ where $\tan \left(\pi-\delta^{\prime}\right)=-0.9286 \sin \delta$. The Yukawa couplings run from the compactification scale down to the electroweak scale in which case the dimensionless Yukawa couplings cannot be used directly for assigning the input mass parameters. But all Yukawa couplings leading to parameters in Eq. (26) are arising from the VEVs of FN singlet fields and we may use those given in Eq. (26) as the input parameters determining the CKM matrix.

\section{Mass matrix in field theory}

Let us present a possibility of obtaining a mass matrix similar to Eq. (26) in field theory. Let the $\mathrm{U}(1)_{\text {anom }} \times \mathrm{U}(1)_{\text {fr }}$ quantum numbers are shown as $\left(Q_{\mathrm{anom}}, Q_{\mathrm{fr}}\right)$. After diagonalizing the $Q_{\mathrm{em}}=\frac{2}{3}$ quark masses, the L- and R-fields of $Q_{\mathrm{em}}=-\frac{1}{3}$ quarks, $\xi$ and $\bar{\eta}$, quantum numbers of $\bar{\eta} \xi$ are

$$
\left.\begin{array}{cccc} 
& \xi_{1 L}(2,-1) & \xi_{2 L}(0,1) & \xi_{3 L}(1,0) \\
\bar{\eta}_{1 R}(1,-1) & (3,-2) & (1,0) & (2,-1) \\
\bar{\eta}_{2 R}(2,1) & (4,0) & (2,2) & (3,1) \\
\bar{\eta}_{3 R}(-1,0) & (1,-1) & (-1,1) & (0,0)
\end{array}\right)
$$

Thus, the quantum numbers of Higgs fields appearing in the mass matrix are

$$
Q\left(M^{d}\right)=\left(\begin{array}{ccc}
(-3,+2) & (-1,0) & (-2,+1) \\
(-4,0) & (-2,-2) & (-3,-1) \\
(-1,+1) & (+1,-1) & (0,0)
\end{array}\right)
$$

To mimic the order appearing in Eq. (26), let us introduce small parameters via the FN SM singlet fields, $\delta_{1}, \delta_{2}, \delta_{3}$, $\Delta_{1}, \Delta_{2}, \epsilon_{1}$, and $\epsilon_{2}$ whose quantum numbers are shown in Table II.

Let us assume that only $\delta_{1}$ and $\Delta_{1}$ have complex VEV's, $\delta_{1} e^{i \delta}$ and $\Delta_{1} e^{i \Delta}$ while all the other FN fields have real VEV's. Thus, $M^{d}$ can be written as

$$
M^{d}=m_{b}\left(\begin{array}{ccc}
\left|\delta_{1}\right|^{3} e^{3 i \delta} \epsilon_{1}^{2} & -\left|\delta_{1}\right| e^{i \delta} & \delta_{2} \epsilon_{1}, \Delta_{2} \epsilon_{1}^{3} \\
-\left|\Delta_{1}\right| e^{i \Delta} & -\left|\Delta_{2}\right| & \left|\Delta_{1}\right| e^{i \Delta} \delta_{3} \epsilon_{2}, \Delta_{2}\left|\delta_{1}\right| e^{i \delta} \epsilon_{1} \\
-\left|\delta_{1}\right| e^{i \delta} \epsilon_{1} & \left(\left|\delta_{1}\right| e^{-i \delta}+\delta_{3}\right) \epsilon_{2} & 1
\end{array}\right),
$$

where the overall constant is $m_{b}$ and for simplicity we do not write group theoretic numbers of $\mathrm{O}(1)$. The element $M_{23}^{d}$ can have $\delta_{1}^{3} e^{3 i \delta} \epsilon_{2}$ which we neglected because it is much smaller than the other terms. A negative signed phase in $M_{32}^{d}$ of Eq. (30) may need a complex conjugated field, but we do not introduce complex conjugated fields in the mass matrix for a SUSY extension.

\section{With SUSY}

Not to introduce complex conjugated fields in the mass matrix, let us consider the fields presented in Table III,

$$
M^{d}=m_{b}\left(\begin{array}{ccc}
\left|\delta_{1}\right|^{3} e^{3 i \delta} \epsilon_{1}^{2}, \Delta_{2}\left|\Delta_{3}\right| e^{i \Delta_{\mathrm{ph} 3}} \epsilon_{1}^{3} & -\left|\delta_{1}\right| e^{i \delta},\left|\Delta_{3}\right| e^{i \Delta_{\mathrm{ph} 3}} \epsilon_{2} & \delta_{1} \Delta_{3}, \Delta_{2} \epsilon_{1}^{3} \\
-\left|\Delta_{1}\right| e^{i \Delta_{\mathrm{ph} 1}} & -\left|\Delta_{2}\right| & \left|\Delta_{1}\right| e^{i \Delta_{\mathrm{ph} 1}} \delta_{3} \epsilon_{2}, \Delta_{2}\left|\delta_{1}\right| e^{i \delta} \epsilon_{1}, \Delta_{2}\left|\Delta_{3}\right| e^{i \Delta_{\mathrm{ph} 3}} \\
-\left|\delta_{1}\right| e^{i \delta} \epsilon_{1} & -\left|\Delta_{3}\right| e^{i \Delta_{3}},-\delta_{3} \epsilon_{2} & 1
\end{array}\right),
$$

For $\delta_{3}=O(1)$ and small $\Delta_{3}$ and $\epsilon_{2}$, and redefine $\xi_{2} \rightarrow \xi_{2} e^{-i \delta}, \bar{\eta}_{2} \rightarrow \bar{\eta}_{2} e^{-i \Delta_{1}}$. By choosing $\Delta_{\mathrm{ph} 1}=\Delta_{\mathrm{ph} 3}=\delta$, and $\delta=\frac{\pi}{2}$, we obtain

$$
M^{d} \simeq m_{b}\left(\begin{array}{ccc}
\mp\left|\delta_{1}\right|^{3} \epsilon_{1}^{2} i & -\left|\delta_{1}\right| & -a_{1}\left|\Delta_{2}\right| \epsilon_{1}^{3} \\
-\left|\Delta_{1}\right| & \left|\Delta_{2}\right| & a_{2}\left|\Delta_{1}\right| \delta_{3} \epsilon_{2}+a_{3} \Delta_{2}\left|\delta_{1}\right| \epsilon_{1}+a_{4} \Delta_{2}\left|\Delta_{3}\right| \\
-\left|\delta_{1}\right| \epsilon_{1} i & -\left|\Delta_{3}\right|+\delta_{3} \epsilon_{2} i & 1
\end{array}\right)
$$

where we introduced $\mathrm{O}(1)$ numbers $a_{1,2,3,4}$. Firstly, $\left|\Delta_{1}\right|=\left|\delta_{1}\right|=4.432 \times 10^{-3}$ and require $a_{1} \Delta_{2} \epsilon_{1}^{3}=0.690 \times 10^{-4}$ (with $a_{1} \simeq 1$ ). Let $\left|\Delta_{2}\right|=0.01918$ and $\left|\delta_{1}\right| \epsilon_{1}=0.9008 \times 10^{-2}$. Then, we have $\epsilon_{1}=2.032, \quad\left|\Delta_{3}\right|=1.557 \times 10^{-2}$, 
TABLE II. FN singlet fields.

\begin{tabular}{lrrrrrrr}
\hline \hline FN fields & $\delta_{1}$ & $\delta_{2}$ & $\delta_{3}$ & $\Delta_{1}$ & $\Delta_{2}$ & $\epsilon_{1}$ & $\epsilon_{2}$ \\
\hline$Q_{\text {anom }}$ & -1 & -2 & +1 & -4 & -2 & 0 & 0 \\
$Q_{\text {fr }}$ & 0 & 0 & 0 & 0 & -2 & +1 & -1 \\
\hline
\end{tabular}

$\delta_{3} \epsilon_{2} \equiv A=3.90 \times 10^{-2} . \quad$ Requiring $\quad a_{2}\left|\Delta_{1}\right| \delta_{3} \epsilon_{2}+$ $a_{3} \Delta_{2}\left|\delta_{1}\right| \epsilon_{1}+a_{4}\left|\Delta_{2}\right|\left|\Delta_{3}\right|=2.99 \times 10^{-4}$, where all term are $\mathrm{O}\left(10^{-4}\right)$. To obtain the relations between phases, $\Delta_{\mathrm{ph} 1}=\Delta_{\mathrm{ph} 3}=\delta$, we can consider the following superpotential,

$$
\begin{aligned}
W_{C P}= & -i \mu_{1} \delta_{1} \delta_{3}+\frac{1}{\mu_{1}} \delta_{1}^{4}+i M_{0}^{2} \Delta_{1}+M_{1} \Delta_{1}^{2}+M_{2} \Delta_{2}^{2} \\
& +i \lambda_{1} \Delta_{1} \Delta_{2}^{2}+i \lambda_{2} \Delta_{3} \delta_{3} \epsilon_{2}+\lambda_{3} \Delta_{2} \Delta_{3}^{2}+\frac{1}{\mu_{2}^{2}} \delta_{1} \Delta_{3}^{3} \epsilon_{1},
\end{aligned}
$$

where parameters are real numbers. The following SUSY conditions lead to the desired relations:
TABLE III. L-handed chiral fields for SUSY extension.

\begin{tabular}{lrrrrrrr}
\hline \hline FN fields & $\delta_{1}$ & $\delta_{3}$ & $\Delta_{1}$ & $\Delta_{2}$ & $\Delta_{3}$ & $\epsilon_{1}$ & $\epsilon_{2}$ \\
\hline$Q_{\text {anom }}$ & -1 & +1 & -4 & -2 & -1 & 0 & 0 \\
$Q_{\text {fr }}$ & 0 & 0 & 0 & -2 & +1 & +1 & -1 \\
\hline \hline
\end{tabular}

$$
\begin{aligned}
\frac{4}{\mu_{1}} \delta_{1}^{3}-i \mu_{1} \delta_{3}+\frac{1}{\mu_{2}^{2}} \Delta_{3}^{3} \epsilon_{1} & =0, \\
-\mu_{1} \delta_{1}+\lambda_{2} \Delta_{3} \epsilon_{2} & =0, \\
i M_{0}^{2}+i \lambda_{1} \Delta_{2}^{2}+2 M_{1} \Delta_{1} & =0, \\
i 2 \lambda_{1} \Delta_{1} \Delta_{2}+\lambda_{3} \Delta_{3}^{2}+2 M_{2} \Delta_{2} & =0, \\
i \lambda_{2} \delta_{3} \epsilon_{2}+2 \lambda_{3} \Delta_{2} \Delta_{3}+\frac{3}{\mu_{2}^{2}} \delta_{1} \Delta_{3}^{2} \epsilon_{1} & =0 .
\end{aligned}
$$

\section{Lepton mass matrices}

Again, we use the KS parametrization [23] to specify the phase $\delta_{L}=\delta_{\mathrm{PMNS}}$ from the $(3,1)$ element of $M_{e}$. Note that the preliminary value $\delta_{\mathrm{PMNS}} \approx-\frac{\pi}{2}$ [24],

$$
U_{\mathrm{PMNS}}=\left(\begin{array}{ccc}
C_{1}, & +S_{1} C_{3}, & +S_{1} S_{3} \\
-C_{2} S_{1}, & +C_{1} C_{2} C_{3}+S_{2} S_{3} e^{-i \delta_{L}}, & +C_{1} C_{2} S_{3}-S_{2} C_{3} e^{-i \delta_{L}} \\
-S_{1} S_{2} e^{i \delta_{L}}, & -C_{2} S_{3}+C_{1} S_{2} C_{3} e^{i \delta_{L},} & +C_{2} C_{3}+C_{1} S_{2} S_{3} e^{i \delta_{L}}
\end{array}\right),
$$

where the parameters are the leptonic parameters, $\Theta_{1,2,3}$ and $\delta_{L}$. Since the PMNS matrix elements are not known as accurately as the CKM matrix elements, we do not present a detail study of the leptonic sector. But note that the phase $\delta_{L}$ in Eq. (35) is the PMNS phase $\delta_{\text {PMNS }}$.

\section{FROM E $\mathrm{E}_{8} \times \mathrm{E}_{8}^{\prime}$ HETEROTIC STRING}

In this section, we attempt to realize the texture of quark mass matrix discussed in Subsection III B. We will not discuss the texture of neutrino mass matrix since the PMNS matrix elements are not known very accurately. Nevertheless, we will comment on the relation of $C P$ phases in the quark and lepton sectors in this section.

\section{A. $Z_{12-I}$ orbifold compactification}

Note that the SM mass matrix

$$
\bar{\Psi}_{I L}^{c} C^{-1} \Psi_{J L} M^{I J}+\text { H.c. }
$$

gives in general non-symmetric mass matrix of $M$ because $\Psi_{L}^{c}$ and $\Psi_{L}$ transform differently under $\mathrm{SU}(2)_{L} \times U(1)_{Y}$. In the GUT model, Majorana neutrinos in the $\mathrm{SU}(2)_{L}$ doublets are embedded in $\mathbf{5}_{0}$ of $\mathrm{SU}(5)$ in the GG model and $\mathbf{5}_{+3}$ in the $\mathrm{SU}(5)_{\text {flip }}$. Then, the effective neutrino mass matrix in these simple GUTs are symmetric. For the quark mass matrix, $\overline{\mathbf{1 0}}_{f} \overline{\mathbf{1 0}}_{f} \overline{\mathbf{5}}_{\mathrm{Higgs}}$ is the up-type quark mass matrix in the GG model, which is symmetric. In the GG model, we usually use diagonalized up-type quark mass matrix, and consider non-symmetric $\mathbf{5}_{f} \overline{\mathbf{1 0}}_{f} \mathbf{5}_{\text {Higgs }}$ for the down-type quark mass matrix. On the other hand, in the $\mathrm{SU}(5)_{\text {flip }}$ the down-type quark mass matrix, $\overline{\mathbf{1 0}}_{f} \overline{\mathbf{1 0}}_{f} \overline{\mathbf{5}}_{\text {Higgs }}$ is symmetric and the up-type quark mass matrix $\mathbf{5}_{f} \overline{\mathbf{1 0}}_{f} \mathbf{5}_{\mathrm{Higgs}}$ is non-symmetric. So, we prefer to consider a symmetric down-type quark mass matrix in $\mathrm{SU}(5)_{\text {flip }}$. The up-type quark mass matrix is non-symmetric, and we can assign different coefficients for $M_{(u) I J}$ and $M_{(u) J I}$,

down type quark mass matrix $=$ symmetric

up type quark mass matrix $=$ asymmetric

The $\mathrm{SU}(5)_{\text {flip }}$ GUT gauge group presented in Ref. [2] is

$$
S U(5) \times U(1)_{X} \times S U(5)^{\prime} \times U(1)^{6},
$$

where, in the notation of [28],

$$
X=(-2,-2,-2,-2,-2,0,0,0)\left(0^{8}\right)^{\prime} .
$$


TABLE IV. The SU $(5)_{\text {flip }}$ fields. Fields needed in the SM are on the four left columns and SM singlet components needed at the GUT scale toward the FN mechanism are on the four right columns. Both neutrino components in $C_{11}$ and $C_{12}$ develop a GUT scale VEV to break $\mathrm{SU}(5)_{\text {flip }}$ down to the SM.

\begin{tabular}{lcccccrr}
\hline \hline $\mathrm{SU}(5)_{\text {flip }}$ (Symbol) & Sect. & $\mathrm{U}(1)_{\text {anom }}\left(Q_{\text {anom }}\right)$ & $Q_{1} / 2$ & $\mathrm{SU}(5)_{\text {flip }}($ Symbol $)$ & Sect. & $\mathrm{U}(1)_{\text {anom }}\left(Q_{\text {anom }}\right)$ & $Q_{1} / 2$ \\
\hline $\mathbf{1}_{-5}\left(S_{1}\right)$ & $U$ & +5 & -3 & $\mathbf{1}_{0}\left(\sigma_{1}\right)$ & $T_{4}^{0}$ & -12 & -4 \\
$\mathbf{1}_{-5}\left(S_{24}^{a}\right)$ & $T_{4}^{0}$ & -3 & -1 & $\mathbf{1}_{0}\left(\sigma_{2}\right)$ & $T_{4}^{0}$ & -2 & -4 \\
$\mathbf{1}_{-5}\left(S_{24}^{b}\right)$ & $T_{4}^{0}$ & -3 & -1 & $\mathbf{1}_{0}\left(\sigma_{3}\right)$ & $T_{4}^{0}$ & -8 & +2 \\
$\overline{\mathbf{1 0}}_{-1}\left(C_{2}\right)$ & $U$ & -13 & -3 & $\mathbf{1}_{0}\left(\sigma_{4}\right)$ & $T_{4}^{0}$ & +10 & +2 \\
$\mathbf{5}_{+3}\left(C_{1}\right)$ & $U$ & -1 & +3 & $\mathbf{1}_{0}\left(\sigma_{5}\right)$ & $T_{6}$ & +14 & 0 \\
$\mathbf{5}_{+3}\left(C_{3 a}\right)$ & $T_{4}^{0}$ & -3 & -1 & $\mathbf{1}_{0}\left(\sigma_{6}\right)$ & $T_{6}$ & -4 & -2 \\
$\mathbf{5}_{+3}\left(C_{3 b}\right)$ & $T_{4}^{0}$ & -3 & -1 & $\mathbf{1}_{0}\left(\sigma_{9}\right)$ & $T_{2}^{0}$ & -6 & -2 \\
$\overline{\mathbf{1 0}}_{-1}\left(C_{4 a}\right)$ & $T_{4}^{0}$ & -3 & -1 & $\mathbf{1}_{0}\left(\sigma_{10}\right)$ & $T_{2}^{0}$ & -6 & +3 \\
$\overline{\mathbf{1 0}}_{-1}\left(C_{4 b}\right)$ & $T_{4}^{0}$ & -3 & -1 & $\mathbf{1}_{0}\left(\sigma_{13}\right)$ & $T_{3}$ & $+\frac{124}{7}$ & +3 \\
$\overline{\mathbf{1 0}}_{-1}\left(C_{11}\right)$ & $T_{3}$ & $-\frac{33}{7}$ & 0 & $\mathbf{1 0}_{+1}\left(C_{12}\right)$ & $T_{9}$ & $+\frac{33}{7}$ & +3 \\
$\mathbf{5}_{-2}\left(H_{u}\right)$ & $T_{6}$ & 0 & 0 & $\mathbf{1}_{0}\left(\sigma_{15}\right)$ & $T_{9}$ & $+\frac{30}{7}$ & +1 \\
$\overline{\mathbf{5}}_{+2}\left(H_{d}\right)$ & $T_{6}$ & 0 & 0 & $\mathbf{1}_{0}\left(\sigma_{21}\right)$ & $T_{1}^{0}$ & $+\frac{12}{7}$ & -1 \\
\hline \hline
\end{tabular}

and six U(1) directions of Ref. [2] are

$$
\begin{aligned}
& Q_{1}=\left(0^{5}, 12,0,0\right)\left(0^{8}\right)^{\prime}, \\
& Q_{2}=\left(0^{5}, 0,12,0\right)\left(0^{8}\right)^{\prime}, \\
& Q_{3}=\left(0^{5}, 0,0,12\right)\left(0^{8}\right)^{\prime}, \\
& Q_{4}=\left(0^{8}\right)\left(0^{4}, 0,12,-12,0\right)^{\prime}, \\
& Q_{5}=\left(0^{8}\right)\left(0^{4}, 0,-6,-6,12\right)^{\prime}, \\
& Q_{6}=\left(0^{8}\right)(-6,-6,-6,-6,18,0,0,6)^{\prime} .
\end{aligned}
$$

$Q_{\text {anom }}$ is given by

$Q_{\mathrm{anom}}=\frac{1}{126}\left(84 Q_{1}+147 Q_{2}-42 Q_{3}-63 Q_{5}-9 Q_{6}\right)$.

We will use notations of Ref. [2] for the names of the fields, twisted sectors $\left(T_{1}^{0}, \ldots, T_{6}\right)$ and the untwisted sector $(U)$. We also list $\frac{1}{2} Q_{1}$ in Table IV such that a discrete subgroup of $U(1)_{1}$ can be used for matter parity if needed. We choose one gauged $\mathrm{U}(1)$ example beyond $\mathrm{U}(1)_{\text {anom }}$, and we checked that any other choice leads to the same conclusion.

\section{B. Doublet-triplet splitting}

In the $\mathrm{SU}(5)_{\text {flip }}$, it is well-known that there is a possibility of doublet-triplet splitting. $C_{12}$ and $C_{14}$ in Eq. (54) develop the GUT scale VEVs,

$$
\begin{aligned}
& \left\langle C_{11}\right\rangle=\left\langle C_{12}\right\rangle \equiv V \\
& \left\langle C_{14}\right\rangle=-V s_{\beta} c_{\gamma}
\end{aligned}
$$

where the first equality is for vanishing D-term at the GUT scale. The renormalizable coupling, including the Higgs quintet $\mathbf{1 0}_{+1} \mathbf{5}_{-2} \mathbf{1 0}_{+1} \sim \Phi^{a b} \Phi^{c} \Phi^{d e} \epsilon_{\text {abcde }}$ might give the GUT scale mass term to colored scalars by $\{d e\}=\{45\}$, but $C_{12} H_{u} C_{12}$ coupling is not allowed by the nonvanishing $Q_{\text {anom }}$. A possible higher dimensional operator consistent with the orbifold selection rules and $\mathrm{U}(1)_{\text {anom }} \times U(1)_{3}$ gauge symmetry is

$$
\begin{aligned}
& \frac{1}{M^{4}} C_{11}\left[\overline{\mathbf{1 0}}_{-1}\left(T_{3}\right)\right] H_{d}\left[\overline{\mathbf{5}}_{+2}\left(T_{6}\right)\right] C_{11}\left[\overline{\mathbf{1 0}}_{-1}\left(T_{3}\right)\right] \sigma_{3}\left[\mathbf{1}\left(T_{4}^{0}\right)\right] \\
& \quad \times \sigma_{5}\left[\mathbf{1}\left(T_{6}\right)\right] \sigma_{21}\left[\mathbf{1}\left(T_{1}^{0}\right)\right] \sigma_{21}\left[\mathbf{1}\left(T_{1}^{0}\right)\right]
\end{aligned}
$$

By giving GUT to Planck scale VEVs to $C_{11}, \sigma_{3}, \sigma_{5}$, and $\sigma_{21}$, we obtain a GUT scale mass term for colored scalars,

$$
M_{T} \epsilon_{\alpha \beta \gamma} \Phi^{\alpha \beta} \Phi^{\gamma}
$$

where $\alpha, \beta, \gamma$ are the color indices. Thus, the color antitriplet in $\overline{\mathbf{1 0}}$ combines with the color triplet in the Higgs quintet $\overline{\mathbf{5}}$. The colored scalar in the Higgs quintet $H_{d}$ is removed at the GUT scale, and there remains just the Higgs doublet from $H_{d}$. For this doublet-triplet splitting, we need

$$
\left\langle\sigma_{3}\right\rangle \neq 0, \quad\left\langle\sigma_{5}\right\rangle \neq 0, \quad\left\langle\sigma_{21}\right\rangle \neq 0,
$$

and the color triplet mass is estimated as

$$
M_{T} \simeq V \frac{\left\langle\sigma_{3}\right\rangle\left\langle\sigma_{5}\right\rangle\left\langle\sigma_{21}\right\rangle^{2}}{M^{4}}
$$


Suppose $V \sim M, \sigma_{3,5} \sim 10^{-2.5} M$ and $\left\langle\sigma_{21}\right\rangle^{2} \sim 10^{-1} M$. Then, we obtain $M_{T} \sim 10^{-6} M \sim 0.6 \times 10^{12} \mathrm{GeV}$ for $M \sim 6 \times 10^{17} \mathrm{GeV}$. $10^{12} \mathrm{GeV}$ colored scalar with small Yukawa couplings of the first family is acceptable. Similarly, considering $\mathrm{C}_{12} \mathrm{H}_{u} \mathrm{C}_{12} \sim \mathbf{1 0}_{+1} \mathbf{5}_{-2} \mathbf{1 0}_{+1}$,

$$
\begin{aligned}
& \frac{1}{M^{4}} C_{12}\left[\mathbf{1 0}_{+1}\left(T_{9}\right)\right] H_{u}\left[\mathbf{5}_{-2}\left(T_{6}\right)\right] C_{12}\left[\mathbf{1 0}_{+1}\left(T_{9}\right)\right] \sigma_{1}\left[\mathbf{1}\left(T_{4}^{0}\right)\right] \\
& \quad \times \sigma_{9}\left[\mathbf{1}\left(T_{2}^{0}\right)\right] \sigma_{15}\left[\mathbf{1}\left(T_{9}\right)\right] \sigma_{15}\left[\mathbf{1}\left(T_{9}\right)\right]
\end{aligned}
$$

the colored scalar in the Higgs quintet $H_{u}$ is removed at the GUT scale and there remains just the Higgs doublet from $H_{u}$. For this, we further require

$$
\left\langle\sigma_{1}\right\rangle \neq 0, \quad\left\langle\sigma_{9}\right\rangle \neq 0, \quad\left\langle\sigma_{15}\right\rangle \neq 0 .
$$

\section{Proton decay problem}

One may consider another gauge symmetry to obtain a $\mathbf{Z}_{2}$ discrete group by breaking $\mathrm{U}(1)_{1}$ by some VEVs of singlet fields carrying even quantum numbers of $Q_{1} / 2$ in Table IV. It can serve as a kind of matter parity since $\mathrm{SU}(5)_{\text {flip }}$ matter fields carry odd $Q_{1} / 2$. But this discrete symmetry does not work because $\left\langle\sigma_{21}\right\rangle$ of Eq. (45) and $\left\langle\sigma_{15}\right\rangle$ of Eq. (48) carry the odd quantum number of $Q_{1} / 2$. We do not have any mechanism for matter parity. The proton decay amplitude must be estimated in detail. ${ }^{5}$

In SUSY models, the dimension 5 proton decay operator must be sufficiently suppressed [50]. The dimension 5 proton decay operators to electronic and muonic leptons are from the superpotential $q^{1} q^{1} q^{1} l^{1,2}$, i.e., $C_{15} C_{15} C_{15} C_{17}$ and $C_{15} C_{15} C_{15} C_{16}$. Note that $C_{15}, C_{17}$, and $C_{16}$ are allowed from the sector $T_{4}^{0}$. Therefore, the $\mathbf{Z}_{12-I}$ orbifold selection rules forbid the product of these four fields from $T_{4}^{0}$, and hence there is no serious proton decay problem from the above dimension 5 operator multiplied by FN singlets ( $\sigma$ 's) appear at least at dimension 7 level in our $\mathbf{Z}_{12-I}$ model.

If it were the GG SU(5), the cubic superpotential written in terms of matter parity violating term, $\overline{\mathbf{1 0}}_{0} \mathbf{5}_{0} \mathbf{5}_{0}$, triggers proton decay as shown in Fig. 1 [51]. In the $\mathrm{SU}(5)_{\text {flip }}$ also there arise dangerous proton decay operators

$$
\overline{\mathbf{1 0}}_{-1}^{m} \overline{\mathbf{1 0}}_{-1}^{m} \mathbf{5}_{+3}^{m} \overline{\mathbf{1 0}}_{-1}^{H}, \mathbf{5}_{+3}^{m} \mathbf{5}_{+3}^{m} \mathbf{1}_{-5}^{m} \overline{\mathbf{1 0}}_{-1}^{H},
$$

where fields with superscript $m$ are matter fields and $\overline{\mathbf{1 0}}_{-1}^{H}$ is the field breaking $\mathrm{SU}(5)_{\text {flip }}$ to the SM. The above operators trigger proton decay in our model by products of $\mathrm{FN}$ singlets $(\sigma$ 's) appear at dimension 10 level,

\footnotetext{
${ }^{5}$ If an R parity is introduced [49], the proton decay problem is automatically solved.
}

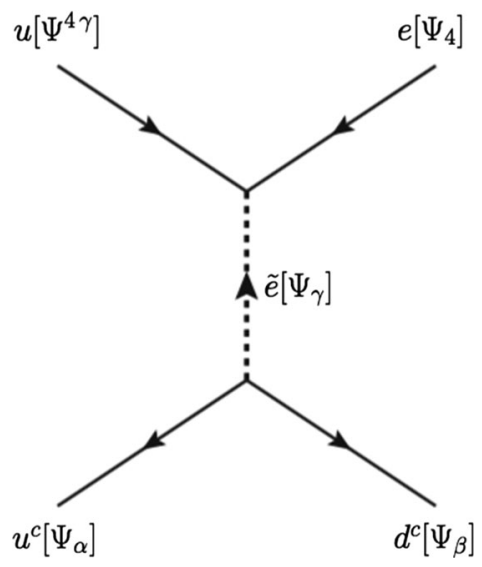

FIG. 1. A diagram for $\Delta B \neq 0$.

$$
\begin{aligned}
& C_{4}\left[\overline{\mathbf{1 0}}_{-1}\left(T_{4}^{0}\right)\right] C_{4}\left[\overline{\mathbf{1 0}}_{-1}\left(T_{4}^{0}\right)\right] C_{3}\left[\mathbf{5}_{+3}\left(T_{4}^{0}\right)\right] \\
& \quad \times \frac{1}{M^{6}}\left\{C_{11}\left[\overline{\mathbf{1 0}}_{-1}\left(T_{3}\right)\right] \sigma_{3}\left[\mathbf{1}\left(T_{4}^{0}\right)\right] \sigma_{3}\left[\mathbf{1}\left(T_{4}^{0}\right)\right]\right. \\
& \left.\quad \times \sigma_{5}\left[\mathbf{1}\left(T_{6}\right)\right] \sigma_{5}\left[\mathbf{1}\left(T_{6}\right)\right] \sigma_{21}\left[\mathbf{1}\left(T_{1}^{0}\right)\right]\right\}, \\
& C_{3}\left[\mathbf{5}_{+3}\left(T_{4}^{0}\right)\right] C_{3}\left[\mathbf{5}_{+3}\left(T_{4}^{0}\right)\right] S_{24}\left[\mathbf{1}_{-5}\left(T_{4}^{0}\right)\right] \\
& \quad \times \frac{1}{M^{6}}\left\{C_{11}\left[\overline{\mathbf{1 0}}_{-1}\left(T_{3}\right)\right] \sigma_{3}\left[\mathbf{1}\left(T_{4}^{0}\right)\right] \sigma_{3}\left[\mathbf{1}\left(T_{4}^{0}\right)\right] \sigma_{5}\left[\mathbf{1}\left(T_{6}\right)\right]\right. \\
& \left.\quad \times \sigma_{5}\left[\mathbf{1}\left(T_{6}\right)\right] \sigma_{21}\left[\mathbf{1}\left(T_{1}^{0}\right)\right]\right\} .
\end{aligned}
$$

All the singlets appearing in Eq. (50) are the needed fields for the doublet-triplet splitting in Eq. (45). The coupling in Fig. 1 is estimated, from the first term of Eq. (50) e.g., as,

$$
\begin{aligned}
& \left\{\frac{1}{M^{6}} C_{11} \sigma_{3}^{2} \sigma_{5}^{2} \sigma_{21}\right\}^{2}\left(M_{\mathrm{SUSY}}\right)^{-2} \\
& \quad \sim \frac{1}{M_{\mathrm{GUT}}{ }^{2}} \frac{C_{11}^{2} \sigma_{3}^{4} \sigma_{5}^{4} \sigma_{21}^{2}}{M^{12}}\left(\frac{M_{\mathrm{GUT}}}{M_{\mathrm{SUSY}}}\right)^{2} .
\end{aligned}
$$

Suppose that the SUSY breaking scale $M_{\text {SUSY }} \sim 10 \mathrm{TeV}$, the GUT scale $M_{\mathrm{GUT}} \approx 3 \times 10^{16} \mathrm{GeV}$, and the compactification scale $M \sim 6 \times 10^{17} \mathrm{GeV}$. Then, the last factor $\sim\left(3 \times 10^{12}\right)^{2}$ is balanced by $M_{\mathrm{vev}} \lesssim 0.5 \times 10^{16} \mathrm{GeV}$ where $M_{\text {vev }}$ is some average VEV of $C_{11}$ and neutral $\sigma$ fields. The estimate given in Eq. (46) can be fitted to this average. Thus, the dimension 6 operator of Fig. 1 can be controlled such that it is not so strong as the dimension 6 operator derived from the exchange of leptoquark gauge bosons in SUSY GUTs.

\section{Families}

There are three $\mathbf{5}_{+3}$ 's and three $\mathbf{1}_{-5}$ 's in Table IV. These include all members of three SM lepton doublets three $u^{c}$-type quarks. However, there are four $\overline{\mathbf{1 0}}_{-1}$ 's in Table IV. So, there are a few possibilities of choosing three SM quark doublets. Out of four $\overline{\mathbf{1 0}}_{-1}$ 's, we always choose $\overline{\mathbf{1 0}}_{-1}$ in the 
$U$ sector. Then, there are three possibilites of choosing two remaining quark doublets: (1) the antisymmetric combination of $\overline{\mathbf{1 0}}_{-1}$ 's from the $T_{4}^{0}$ sector and $\overline{\mathbf{1 0}}_{-1}$ from the $T_{3}$ sector, (2) two $\overline{\mathbf{1 0}}_{-1}$ 's from the $T_{4}^{0}$ sector, and (3) a linear combination of $\overline{\mathbf{1 0}}_{-1}$ of $T_{3}$ and antisymmetric $\overline{\mathbf{1 0}}_{-1}$ from $T_{4}^{0}$, and a linear combination of $\overline{\mathbf{1 0}}_{-1}$ of $T_{3}$ and symmetric $\overline{\mathbf{1 0}}_{-1}$ from $T_{4}^{0}$. All these are considered by mixing three $\overline{\mathbf{1 0}}_{-1}$ 's, introducing three angles $\alpha, \beta$ and $\gamma$,

$$
\begin{aligned}
C_{13}\left[\overline{\mathbf{1 0}}_{-1}\left(T_{4}^{0}\right), \overline{\mathbf{1 0}}_{-1}\left(T_{3}\right)\right] \equiv & +C_{11} c_{\beta}+C_{4 a} c_{\alpha} s_{\beta}-C_{4 b} s_{\alpha} s_{\beta}, \\
C_{14}\left[\overline{\mathbf{1 0}}_{-1}\left(T_{4}^{0}\right), \overline{\mathbf{1 0}}_{-1}\left(T_{3}\right)\right] \equiv & -C_{11} s_{\beta} c_{\gamma}+C_{4 a}\left(c_{\alpha} c_{\beta} c_{\gamma}-s_{\alpha} s_{\gamma}\right) \\
& -C_{4 b}\left(s_{\alpha} c_{\beta} c_{\gamma}+c_{\alpha} s_{\gamma}\right), \\
C_{15}\left[\overline{\mathbf{1 0}}_{-1}\left(T_{4}^{0}\right), \overline{\mathbf{1 0}}_{-1}\left(T_{3}\right)\right] \equiv & -C_{11} s_{\beta} s_{\gamma}+C_{4 a}\left(s_{\alpha} c_{\gamma}+c_{\alpha} c_{\beta} s_{\gamma}\right) \\
& +C_{4 b}\left(c_{\alpha} c_{\gamma}-s_{\alpha} c_{\beta} s_{\gamma}\right) .
\end{aligned}
$$

where $s_{\alpha, \beta, \gamma}=\sin _{\alpha, \beta, \gamma}$ and $c_{\alpha, \beta, \gamma}=\cos _{\alpha, \beta, \gamma}$. We choose two out of the above three combinations. Similarly, we define

$$
\begin{aligned}
& C_{16}\left[\mathbf{5}_{+3}\left(T_{4}^{0}\right)\right] \equiv \frac{1}{\sqrt{2}}\left(+C_{3 a}+C_{3 b}\right), \\
& C_{17}\left[\mathbf{5}_{+3}\left(T_{4}^{0}\right)\right] \equiv \frac{1}{\sqrt{2}}\left(-C_{3 a}+C_{3 b}\right) .
\end{aligned}
$$

Now, let us identify $\mathbf{1 0}_{+1}$ and $\overline{\mathbf{1 0}}_{-1}$ 's of Table I as

$$
C_{12}\left[\mathbf{1 0}_{+1}\right] \oplus C_{14}\left[\overline{\mathbf{1 0}}_{-1}\right]:
$$

The Higgs set for breaking $S U(5) \times U(1)_{X}$,

and

$$
\begin{aligned}
& C_{15}: 1 \text { st family, } \\
& C_{13}: 2 \text { nd family, } \\
& C_{2}: 3 \text { rd family, }
\end{aligned}
$$

and $\mathbf{5}_{+3}$ 's of Table I as

$$
\begin{aligned}
& C_{17}: 1 \text { st family, } \\
& C_{16}: 2 \text { nd family, } \\
& C_{1}: 3 \text { rd family, }
\end{aligned}
$$

In this paper, it is outside the scope of current analysis to see the details of superpotential. So, we choose the needed VEVs by hand.

\section{Down-type quarks}

Let us scale scalar fields and mass matrices such that they are made dimensionless by dividing with a mass parameter, e.g., by $M$.

The down-type quark masses are

$$
\begin{aligned}
& M_{d(11)}^{w}=C_{15}\left[\overline{\mathbf{1 0}}_{T_{3}}\right] C_{15}\left[\overline{\mathbf{1 0}}_{T_{3}}\right] H_{d}\left(\overline{\mathbf{5}}_{T_{6}}\right) \sigma_{1}\left(\mathbf{1}_{T_{4}^{0}}\right) \sigma_{6}\left(\mathbf{1}_{T_{6}}\right) \sigma_{6}\left(\mathbf{1}_{T_{6}}\right) \sigma_{9}\left(\mathbf{1}_{T_{2}^{0}}\right) \sigma_{13}\left(\mathbf{1}_{T_{3}}\right) \sigma_{13}\left(\mathbf{1}_{T_{3}}\right), \\
& M_{d(22)}^{w}=C_{13}\left(\overline{\mathbf{1 0}}_{T_{4}^{0}}\right) C_{13}\left(\overline{\mathbf{1 0}}_{T_{4}^{0}}\right) H_{d}\left(\overline{\mathbf{5}}_{T_{6}}\right)\left\{\sigma_{4}\left(\mathbf{1}_{T_{4}^{0}}\right) \sigma_{6}\left(\mathbf{1}_{T_{6}}\right), \sigma_{15}\left(\mathbf{1}_{T_{9}}\right) \sigma_{21}\left(\mathbf{1}_{T_{1}^{0}}\right)\right\} \\
& M_{d(33)}^{w}=C_{2}\left(\overline{\mathbf{1 0}}_{U}\right) C_{2}\left(\overline{\mathbf{1 0}}_{U}\right) H_{d}\left(\overline{\mathbf{5}}_{T_{6}}\right) \sigma_{5}\left(\mathbf{1}_{T_{6}}\right) \sigma_{3}\left(\mathbf{1}_{T_{4}^{0}}\right) \sigma_{4}\left(\mathbf{1}_{T_{4}^{0}}\right) \sigma_{4}\left(\mathbf{1}_{T_{4}^{0}}\right),
\end{aligned}
$$

where we presesented only the antisymmetric part in $M_{d(22)}^{w}$ and only the component from $T_{3}$ in $M_{d(11)}^{w}$. For the down-type quarks, it is enough to show nonzero $M_{d(33)}^{w}$ and $M_{d(22)}^{w}$ and the conditions for making the off-diagonal elements vanish,

$$
\begin{aligned}
& M_{d(12)}^{w}\left.=C_{15}\left(\overline{\mathbf{1 0}}_{T_{4}^{0}}\right) C_{13}\left[\overline{\mathbf{1 0}}_{T_{3}}\right] H_{d}\left(\overline{\mathbf{5}}_{T_{6}}\right)\right)\left\{\sigma_{2}\left(\mathbf{1}_{T_{4}^{0}}\right) \sigma_{3}\left(\mathbf{1}_{T_{4}^{0}}\right) \sigma_{13}\left(\mathbf{1}_{T_{3}}\right), \sigma_{6}\left(\mathbf{1}_{T_{6}}\right) \sigma_{9}\left(\mathbf{1}_{T_{2}^{0}}\right) \sigma_{13}\left(\mathbf{1}_{T_{3}}\right)\right\}=0, \\
& M_{d(21)}^{w}=C_{13}\left[\overline{\mathbf{1 0}}_{T_{3}}\right] C_{15}\left(\overline{\mathbf{1 0}}_{T_{4}^{0}}\right) H_{d}\left(\overline{\mathbf{5}}_{T_{6}}\right)\left\{\sigma_{2}\left(\mathbf{1}_{T_{4}^{0}}\right) \sigma_{3}\left(\mathbf{1}_{T_{4}^{0}}\right) \sigma_{13}\left(\mathbf{1}_{T_{3}}\right), \sigma_{6}\left(\mathbf{1}_{T_{6}}\right) \sigma_{9}\left(\mathbf{1}_{T_{2}^{0}}\right) \sigma_{13}\left(\mathbf{1}_{T_{3}}\right)\right\}=0, \\
& M_{d(13)}^{w}=C_{15}\left(\overline{\mathbf{1 0}}_{T_{4}^{0}}\right) C_{2}\left(\overline{\mathbf{1 0}}_{U}\right) H_{d}\left(\overline{\mathbf{5}}_{T_{6}}\right)\left\{c \sigma_{4}\left(\mathbf{1}_{T_{4}^{0}}\right) \sigma_{4}\left(\mathbf{1}_{T_{4}^{0}}\right) \sigma_{6}\left(\mathbf{1}_{T_{6}}\right)+c^{\prime} \sigma_{4}\left(\mathbf{1}_{T_{4}^{0}}\right) \sigma_{15}\left(\mathbf{1}_{T_{9}}\right) \sigma_{21}\left(\mathbf{1}_{T_{1}^{0}}\right)\right\}=0, \\
& M_{d(31)}^{w}=C_{2}\left(\overline{\mathbf{1 0}}_{U}\right) C_{15}\left(\overline{\mathbf{1 0}}_{T_{4}^{0}}\right) H_{d}\left(\overline{\mathbf{5}}_{T_{6}}\right)\left\{c \sigma_{4}\left(\mathbf{1}_{T_{4}^{0}}\right) \sigma_{4}\left(\mathbf{1}_{T_{4}^{0}}\right) \sigma_{6}\left(\mathbf{1}_{T_{6}}\right)+c^{\prime} \sigma_{4}\left(\mathbf{1}_{T_{4}^{0}}\right) \sigma_{15}\left(\mathbf{1}_{T_{9}}\right) \sigma_{21}\left(\mathbf{1}_{T_{1}^{0}}\right\}=0,\right. \\
& M_{d(23)}^{w}=C_{13}\left[\overline{\mathbf{1 0}}_{T_{3}}\right] C_{2}\left(\overline{\mathbf{1 0}}_{U}\right) H_{d}\left(\overline{\mathbf{5}}_{T_{6}}\right) \sigma_{13}\left(\mathbf{1}_{T_{3}}\right)=0, \\
& M_{d(32)}^{w}=C_{2}\left(\overline{\mathbf{1 0}}_{U}\right) C_{13}\left[\overline{\mathbf{1 0}}_{T_{3}}\right] H_{d}\left(\overline{\mathbf{5}}_{T_{6}}\right) \sigma_{13}\left(\mathbf{1}_{T_{3}}\right)=0 .
\end{aligned}
$$

To satisfy the conditions of Eq. (58), let us choose

$$
\left\langle\sigma_{13}\right\rangle=0
$$


and

$$
c\left\langle\sigma_{4} \sigma_{6}\right\rangle+c^{\prime}\left\langle\sigma_{15} \sigma_{21}\right\rangle=0 .
$$

$M_{d(13)}^{w}$ and $M_{d(31)}^{w}$ can be made to vanish.

\section{Up-type quarks}

Therefore, we consider the $W_{\mu}^{-}$coupling instead of $W_{\mu}^{+}$coupling of Eq. (24), $V_{u}^{\dagger} M_{u}^{\mathrm{diag}} U_{u}$ as

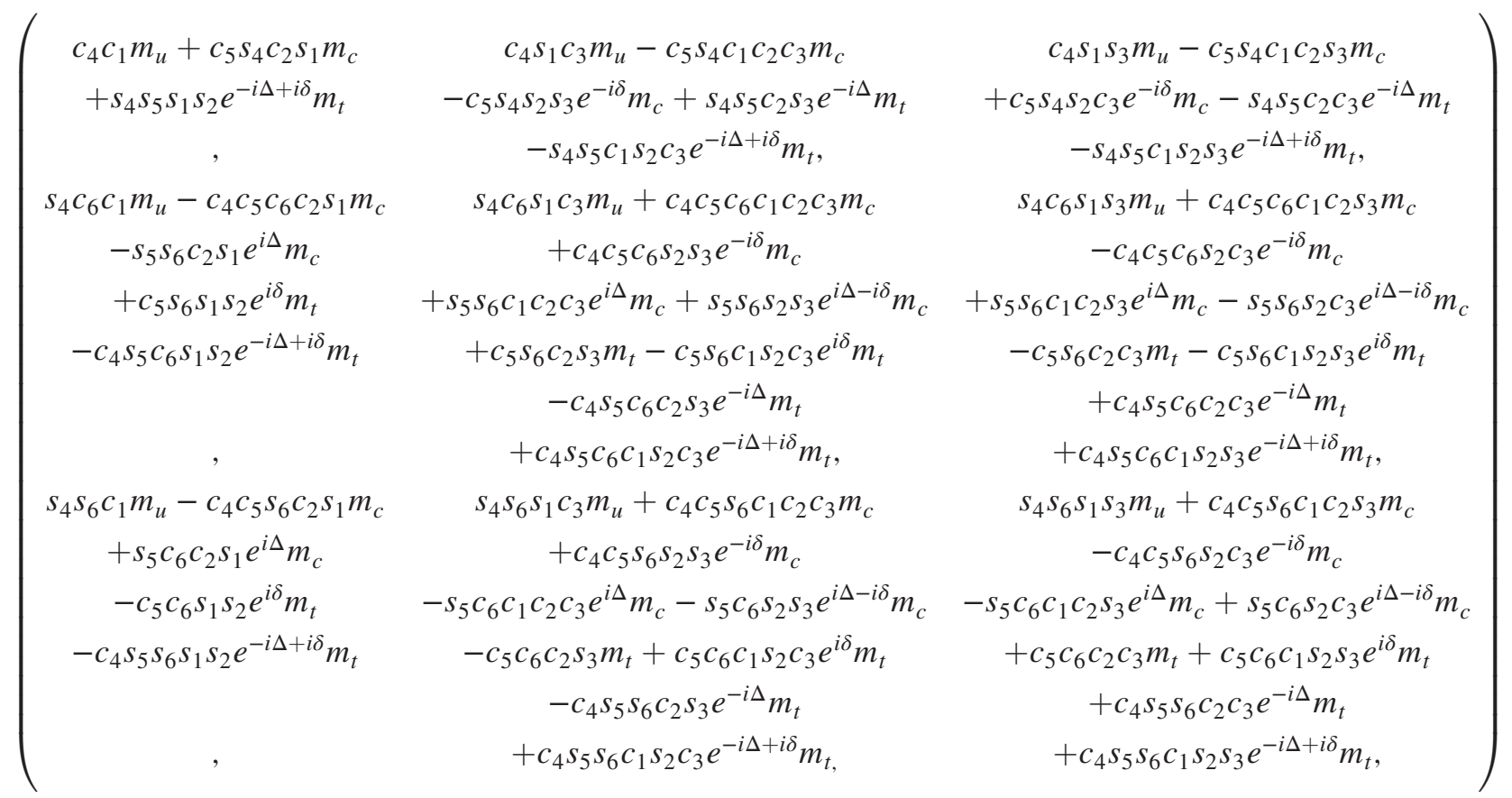

Change the sign $m_{u} \rightarrow-m_{u}$, and to reduce the number of parameters let us choose parameters of R-fields as

$$
\frac{s_{4}}{c_{4}}=\frac{s_{1}}{c_{1}}, \quad \frac{s_{5}}{c_{5}}=\frac{m_{c}}{m_{t}} \frac{s_{2}}{c_{2}}, \quad s_{6}=0, \quad \Delta=\delta .
$$

Then, we obtain

$$
V_{u}^{\dagger} M_{u}^{\mathrm{diag}} U_{u}=\left(\begin{array}{ccc}
-c_{4} c_{1} m_{u}+c_{5} s_{4} c_{2}^{-1} s_{1} m_{c}, & -c_{4} s_{1} c_{3} m_{u}-c_{5} s_{4} c_{1} c_{2}^{-1} c_{3} m_{c}, & -c_{4} s_{1} s_{3} m_{u}-c_{5} c_{4} s_{1} c_{2}^{-1} s_{3} m_{c} \\
-s_{4} c_{1} m_{u} & -s_{4} s_{1} c_{3} m_{u} & -s_{4} s_{1} s_{3} m_{u} \\
-c_{4} c_{5} s_{1} c_{2}^{-1} m_{c}, & +c_{4} c_{5} c_{1} c_{3} c_{2}^{-1} m_{c}, & +c_{4} c_{5} c_{1} s_{3} c_{2}^{-1} m_{c}, \\
+s_{5} c_{2} s_{1} e^{i \delta} m_{c} & -s_{5} c_{1} c_{2} c_{3} e^{i \delta} m_{c}-s_{5} s_{2} s_{3} m_{c} & -s_{5} c_{1} c_{2} s_{3} e^{i \delta} m_{c}+s_{5} s_{2} c_{3} m_{c} \\
-c_{5} s_{1} s_{2} e^{i \delta} m_{t}, & -c_{5} c_{2} s_{3} m_{t}+c_{5} c_{1} s_{2} c_{3} e^{i \delta} m_{t}, & +c_{5} c_{2} c_{3} m_{t}+c_{5} c_{1} s_{2} s_{3} e^{i \delta} m_{t},
\end{array}\right)
$$

where we require $c_{2}, c_{3}, c_{5} \simeq \mathrm{O}(1)$. Also, $s_{5}$ can be $\mathrm{O}(1)$. Thus, we consider,

$$
V_{u}^{\dagger} M_{u}^{\mathrm{diag}} U_{u}=m_{t}\left(\begin{array}{ccc}
-c_{4} c_{1} \frac{m_{u}}{c_{5} m_{t}}+s_{4} s_{1} \frac{m_{c}}{m_{t}}, & -c_{4} s_{1} \frac{m_{u}}{c_{5} m_{t}}-s_{4} c_{1} \frac{m_{c}}{m_{t}}, & -c_{4} s_{1} s_{3} \frac{m_{c}}{m_{t}} \\
-s_{4} c_{1} \frac{m_{u}}{c_{5} m_{t}}-c_{4} s_{1} \frac{m_{c}}{m_{t}}, & +c_{4} c_{1} \frac{m_{c}}{m_{t}}, & +c_{4} c_{1} s_{3} \frac{m_{c}}{m_{t}} \\
-s_{1}\left(s_{2}-s_{5} c_{2} \frac{m_{c}}{c_{5} m_{t}}\right) e^{i \delta \frac{m_{c}}{m_{t}}}, & {\left[-s_{3}+c_{1}\left(s_{2}-s_{5} c_{3} \frac{m_{c}}{c_{5} m_{t}}\right) e^{i \delta}\right] \frac{m_{c}}{m_{t}},} & 1
\end{array}\right)
$$

where we neglected $m_{t} s_{2} s_{3}, m_{c} s_{2}, m_{c} s_{3}$. 


$$
\begin{gathered}
s_{1}=0.2252, \quad c_{1}=0.9743, \quad s_{2}=0.0400, \quad c_{2}=0.9992, \quad s_{3}=0.01557, \quad c_{3}=0.9999, \\
m_{u}=2.5 \mathrm{MeV}, \quad m_{c}=1280 \mathrm{MeV}, \quad m_{t}=173 \mathrm{GeV}, \quad \frac{m_{u}}{m_{t}}=1.45 \times 10^{-5}, \quad \frac{m_{c}}{m_{t}}=0.74 \times 10^{-2},
\end{gathered}
$$

so that $M_{u}^{w} / m_{t}$ is approximately given by

$$
\left(\begin{array}{ccc}
+1.67 \times 10^{-3} s_{4}, & -0.721 \times 10^{-2} s_{4}, & -2.59 \times 10^{-5} c_{4} \\
-1.67 \times 10^{-3} c_{4}, & +0.721 \times 10^{-2} c_{4}, & +1.12 \times 10^{-4} c_{4} \\
\left(-0.67 \times 10^{-6}+1.24 \times 10^{-5} t_{5}\right) e^{i \delta}, & -1.15 \times 10^{-3}+\left(2.88 \times 10^{-4}-0.534 \times 10^{-4} t_{5}\right) e^{i \delta}, & 1
\end{array}\right)
$$

The up-type quark masses are

$$
\begin{aligned}
& M_{u(11)}^{w}=0, \\
& M_{u(22)}^{w}=C_{16}\left(\mathbf{5}_{T_{4}^{0}}\right) C_{13}\left(\overline{\mathbf{1 0}}_{T_{4}^{0}}, \overline{\mathbf{1 0}}_{T_{3}}\right) H_{u}\left(\mathbf{5}_{T_{6}}\right)\left\{\sigma_{4}\left(T_{4}^{0}\right) \sigma_{6}\left(T_{6}\right), \sigma_{15}\left(T_{9}\right) \sigma_{21}\left(T_{1}^{0}\right) ; \sigma_{4}\left(T_{4}^{0}\right) \sigma_{6}\left(T_{6}\right) \sigma_{21}\left(T_{1}^{0}\right)\right\}, \\
& M_{u(33)}^{w}=C_{1}\left(\mathbf{5}_{U}\right) C_{2}\left(\overline{\mathbf{1 0}}_{U}\right) H_{u}\left(\mathbf{5}_{T_{6}}\right) \sigma_{5}\left(\mathbf{1}_{T_{6}}\right), \\
& M_{u(12)}^{w}=C_{17}\left(\mathbf{5}_{T_{4}^{0}}\right) C_{13}\left(\overline{\mathbf{1 0}}_{T_{4}^{0}}, \overline{\mathbf{1 0}}_{T_{3}}\right) H_{u}\left(\mathbf{5}_{T_{6}}\right)\left\{\sigma_{3}\left(T_{4}^{0}\right) \sigma_{5}\left(T_{6}\right), \sigma_{15}\left(T_{9}\right) \sigma_{21}\left(T_{1}^{0}\right) ;\right. \\
& \left.\sigma_{2}\left(\mathbf{1}_{T_{4}^{0}}\right) \sigma_{3}\left(\mathbf{1}_{T_{4}^{0}}\right) \sigma_{13}\left(\mathbf{1}_{T_{3}}\right), \sigma_{6}\left(\mathbf{1}_{T_{6}}\right) \sigma_{9}\left(\mathbf{1}_{T_{2}^{0}}\right) \sigma_{13}\left(\mathbf{1}_{T_{3}}\right), \sigma_{21}\left(T_{1}^{0}\right) \sigma_{3}\left(T_{4}^{0}\right) \sigma_{5}\left(T_{6}\right)\right\}, \\
& M_{u(21)}^{w}=C_{16}\left(\mathbf{5}_{T_{4}^{0}}\right) C_{15}\left(\overline{\mathbf{1 0}}_{T_{4}^{0}}, \overline{\mathbf{1 0}}_{T_{3}}\right) H_{u}\left(\mathbf{5}_{T_{6}}\right)\left\{\sigma_{3}\left(T_{4}^{0}\right) \sigma_{5}\left(T_{6}\right), \sigma_{15}\left(T_{9}\right) \sigma_{21}\left(T_{1}^{0}\right) ;\right. \\
& \left.\sigma_{2}\left(\mathbf{1}_{T_{4}^{0}}\right) \sigma_{3}\left(\mathbf{1}_{T_{4}^{0}}\right) \sigma_{13}\left(\mathbf{1}_{T_{3}}\right), \sigma_{6}\left(\mathbf{1}_{T_{6}}\right) \sigma_{9}\left(\mathbf{1}_{T_{2}^{0}}\right) \sigma_{13}\left(\mathbf{1}_{T_{3}}\right), \sigma_{21}\left(T_{1}^{0}\right) \sigma_{3}\left(T_{4}^{0}\right) \sigma_{5}\left(T_{6}\right)\right\}, \\
& M_{u(23)}^{w}=C_{16}\left(\mathbf{5}_{T_{4}^{0}}\right) C_{2}\left(\overline{\mathbf{1 0}}_{U}\right) H_{u}\left(\mathbf{5}_{T_{6}}\right) \sigma_{3}\left(\mathbf{1}_{T_{4}^{0}}\right) \sigma_{4}\left(\mathbf{1}_{T_{4}^{0}}\right) \sigma_{5}\left(\mathbf{1}_{T_{6}}\right), \\
& M_{u(32)}^{w}=C_{1}\left(\mathbf{5}_{U}\right) C_{13}\left(\overline{\mathbf{1 0}}_{T_{4}^{0}}, \overline{\mathbf{1 0}}_{T_{3}}\right) H_{u}\left(\mathbf{5}_{T_{6}}\right)\left\{\sigma_{5}\left(\mathbf{1}_{T_{6}}\right) \sigma_{6}\left(\mathbf{1}_{T_{6}}\right) \sigma_{9}\left(\mathbf{1}_{T_{2}^{0}}\right), \sigma_{2}\left(\mathbf{1}_{T_{4}^{0}}\right) \sigma_{4}\left(\mathbf{1}_{T_{4}^{0}}\right) \sigma_{6}\left(\mathbf{1}_{T_{6}}\right) ;\right. \\
& \sigma_{13}\left(\mathbf{1}_{T_{3}}\right) \sigma_{2}\left(\mathbf{1}_{T_{4}^{0}}\right) \sigma_{6}\left(\mathbf{1}_{T_{6}}\right) \sigma_{9}\left(\mathbf{1}_{T_{2}^{0}}\right), \sigma_{13}\left(\mathbf{1}_{T_{3}}\right) \sigma_{2}\left(\mathbf{1}_{T_{4}^{0}}\right)^{2} \sigma_{3}\left(\mathbf{1}_{T_{4}^{0}}\right), \sigma_{21}\left(\mathbf{1}_{T_{1}^{0}}\right) \sigma_{2}\left(\mathbf{1}_{T_{4}^{0}}\right) \sigma_{3}\left(\mathbf{1}_{T_{4}^{0}}\right) \sigma_{5}\left(\mathbf{1}_{T_{6}}\right) ; \\
& \left.\sigma_{21}\left(\mathbf{1}_{T_{1}^{0}}\right) \sigma_{5}\left(\mathbf{1}_{T_{6}}\right) \sigma_{6}\left(\mathbf{1}_{T_{6}}\right) \sigma_{9}\left(\mathbf{1}_{T_{2}^{0}}\right), \sigma_{2}\left(\mathbf{1}_{T_{4}^{0}}\right) \sigma_{15}\left(\mathbf{1}_{T_{9}}\right) \sigma_{21}\left(\mathbf{1}_{T_{1}^{0}}\right)^{2}\right\} \\
& M_{u(13)}^{w}=0 \\
& M_{u(31)}^{w}=C_{1}\left(\mathbf{5}_{U}\right) C_{13}\left(\overline{\mathbf{1 0}}_{T_{4}^{0}}, \overline{\mathbf{1 0}}_{T_{3}}\right) H_{u}\left(\mathbf{5}_{T_{6}}\right)\left\{\sigma_{5}\left(\mathbf{1}_{T_{6}}\right) \sigma_{6}\left(\mathbf{1}_{T_{6}}\right) \sigma_{9}\left(\mathbf{1}_{T_{2}^{0}}\right), \sigma_{2}\left(\mathbf{1}_{T_{4}^{0}}\right) \sigma_{4}\left(\mathbf{1}_{T_{4}^{0}}\right) \sigma_{6}\left(\mathbf{1}_{T_{6}}\right) ;\right. \\
& \sigma_{13}\left(\mathbf{1}_{T_{3}}\right) \sigma_{2}\left(\mathbf{1}_{T_{4}^{0}}\right) \sigma_{6}\left(\mathbf{1}_{T_{6}}\right) \sigma_{9}\left(\mathbf{1}_{T_{2}^{0}}\right), \sigma_{13}\left(\mathbf{1}_{T_{3}}\right) \sigma_{2}\left(\mathbf{1}_{T_{4}^{0}}\right)^{2} \sigma_{3}\left(\mathbf{1}_{T_{4}^{0}}\right), \sigma_{21}\left(\mathbf{1}_{T_{1}^{0}}\right) \sigma_{2}\left(\mathbf{1}_{T_{4}^{0}}\right) \sigma_{3}\left(\mathbf{1}_{T_{4}^{0}}\right) \sigma_{5}\left(\mathbf{1}_{T_{6}}\right) ; \\
& \left.\sigma_{21}\left(\mathbf{1}_{T_{1}^{0}}\right) \sigma_{5}\left(\mathbf{1}_{T_{6}}\right) \sigma_{6}\left(\mathbf{1}_{T_{6}}\right) \sigma_{9}\left(\mathbf{1}_{T_{2}^{0}}\right), \sigma_{2}\left(\mathbf{1}_{T_{4}^{0}}\right) \sigma_{15}\left(\mathbf{1}_{T_{9}}\right) \sigma_{21}\left(\mathbf{1}_{T_{1}^{0}}\right)^{2}\right\} \text {. }
\end{aligned}
$$

$M_{u(33)}^{w}$ is the largest value, and we set $\left\langle\sigma_{5}\right\rangle=O(1)$, and automatically we have $M_{u(11)}^{w}=M_{u(13)}^{w}=0$ by the unavoidable antisymmetric property among $\mathbf{5}_{-3}\left(T_{4}^{0}\right)$, viz. $C_{17}$ in Eq. (53).

The following example is just showing a possibility. We have chosen $\sigma_{13}=0$ in Eq. (59) to make down-type quark masses diagonal. Let us further simplify by setting $\left\langle\sigma_{2}\right\rangle=0$,

$$
\begin{aligned}
M_{u(11)}^{w}= & 0 \\
M_{u(22)}^{w}= & C_{16}\left(\mathbf{5}_{T_{4}^{0}}\right) C_{13}\left(\overline{\mathbf{1 0}}_{T_{4}^{0}}, \overline{\mathbf{1 0}}_{T_{3}}\right) H_{u}\left(\mathbf{5}_{T_{6}}\right)\left\{\frac{1}{\sigma_{5}\left(T_{6}\right)} \sigma_{4}\left(T_{4}^{0}\right) \sigma_{6}\left(T_{6}\right), \frac{\sigma_{15}\left(T_{9}\right)}{\sigma_{5}\left(T_{6}\right)} \sigma_{21}\left(T_{1}^{0}\right) ;\right. \\
& \left.\frac{\sigma_{15}\left(T_{9}\right)}{\sigma_{5}\left(T_{6}\right)} \sigma_{21}\left(T_{1}^{0}\right)^{2}, \sigma_{3}\left(T_{4}^{0}\right) \sigma_{21}\left(T_{1}^{0}\right)\right\} \sigma_{5}\left(T_{6}\right), \\
M_{u(33)}^{w}= & C_{1}\left(\mathbf{5}_{U}\right) C_{2}\left(\overline{\mathbf{1 0}}_{U}\right) H_{u}\left(\mathbf{5}_{T_{6}}\right) \sigma_{5}\left(\mathbf{1}_{T_{6}}\right)
\end{aligned}
$$




$$
\begin{aligned}
& M_{u(12)}^{w}=C_{17}\left(\mathbf{5}_{T_{4}^{0}}\right) C_{13}\left(\overline{\mathbf{1 0}}_{T_{4}^{0}}, \overline{\mathbf{1 0}}_{T_{3}}\right) H_{u}\left(\mathbf{5}_{T_{6}}\right)\left\{\sigma_{3}\left(T_{4}^{0}\right), \frac{1}{\sigma_{5}\left(T_{6}\right)} \sigma_{15}\left(T_{9}\right) \sigma_{21}\left(T_{1}^{0}\right) ; 0\right\} \sigma_{5}\left(T_{6}\right), \\
& M_{u(21)}^{w}=C_{16}\left(\mathbf{5}_{T_{4}^{0}}\right) C_{15}\left(\overline{\mathbf{1 0}}_{T_{4}^{0}}, \overline{\mathbf{1 0}}_{T_{3}}\right) H_{u}\left(\mathbf{5}_{T_{6}}\right)\left\{\sigma_{3}\left(T_{4}^{0}\right), \frac{1}{\sigma_{5}\left(T_{6}\right)}, \sigma_{15}\left(T_{9}\right) \sigma_{21}\left(T_{1}^{0}\right) ; \sigma_{21}\left(T_{1}^{0}\right) \sigma_{3}\left(T_{4}^{0}\right)\right\} \sigma_{5}\left(T_{6}\right), \\
& M_{u(23)}^{w}=C_{16}\left(\mathbf{5}_{T_{4}^{0}}\right) C_{2}\left(\overline{\mathbf{1 0}}_{U}\right) H_{u}\left(\mathbf{5}_{T_{6}}\right)\left\{\sigma_{3}\left(\mathbf{1}_{T_{4}^{0}}\right) \sigma_{4}\left(\mathbf{1}_{T_{4}^{0}}\right)\right\} \sigma_{5}\left(\mathbf{1}_{T_{6}}\right), \\
& M_{u(32)}^{w}=C_{1}\left(\mathbf{5}_{U}\right) C_{13}\left(\overline{\mathbf{1 0}}_{T_{4}^{0}}, \overline{\mathbf{1 0}}_{T_{3}}\right) H_{u}\left(\mathbf{5}_{T_{6}}\right)\left\{0 ; \sigma_{6}\left(\mathbf{1}_{T_{6}}\right) \sigma_{9}\left(\mathbf{1}_{T_{2}^{0}}\right) \sigma_{21}\left(\mathbf{1}_{T_{1}^{0}}\right)\right\} \sigma_{5}\left(\mathbf{1}_{T_{6}}\right), \\
& M_{u(13)}^{w}=0 \\
& M_{u(31)}^{w}=C_{1}\left(\mathbf{5}_{U}\right) C_{15}\left(\overline{\mathbf{1 0}}_{T_{4}^{0}}, \overline{\mathbf{1 0}}_{T_{3}}\right) H_{u}\left(\mathbf{5}_{T_{6}}\right)\left\{\sigma_{6}\left(\mathbf{1}_{T_{6}}\right) \sigma_{9}\left(\mathbf{1}_{T_{2}^{0}}\right) ; \sigma_{6}\left(\mathbf{1}_{T_{6}}\right) \sigma_{9}\left(\mathbf{1}_{T_{2}^{0}}\right) \sigma_{21}\left(\mathbf{1}_{T_{1}^{0}}\right)\right\} \sigma_{5}\left(\mathbf{1}_{T_{6}}\right) .
\end{aligned}
$$

where the antisymmetric combination of $\overline{\mathbf{1 0}}_{-1}$ 's from $T_{4}^{0}$ is written before the semicolon and the symmetric combinations of $\overline{\mathbf{1 0}}_{-1}$ 's from $T_{4}^{0}$ is written after the semicolon. Zeros indicate this symmetry properties.

$$
\left(\begin{array}{ccc}
0, & \left(p \sigma_{3}+q \frac{\sigma_{15} \sigma_{21}}{\sigma_{5}}\right), & 0 \\
\left(c \sigma_{3}+d \frac{\sigma_{15} \sigma_{21}}{\sigma_{5}}\right)+e \sigma_{3} \sigma_{21}, & \left.f \frac{\sigma_{4} \sigma_{6}}{\sigma_{5}}+g \frac{\sigma_{15} \sigma_{21}}{\sigma_{5}}+h \frac{\sigma_{15} \sigma_{21}^{2}}{\sigma_{5}}+k \sigma_{3} \sigma_{21}\right), & \ell \sigma_{3} \sigma_{4} \\
a \sigma_{6} \sigma_{9}\left(1+r \sigma_{21}\right), & b \sigma_{6} \sigma_{9} \sigma_{21}, & 1
\end{array}\right)
$$

To present a simple numerics, let us neglect the $\sigma_{15}$ terms. So, consider

$$
\left(\begin{array}{ccc}
0, & p \sigma_{3}, & 0 \\
c \sigma_{3}+e \sigma_{3} \sigma_{21}, & f \frac{\sigma_{4} \sigma_{6}}{\sigma_{5}}+k \sigma_{3} \sigma_{21}, & \ell \sigma_{3} \sigma_{4} \\
a \sigma_{6} \sigma_{9}\left(1+r \sigma_{21}\right), & b \sigma_{6} \sigma_{9} \sigma_{21}, & 1
\end{array}\right)
$$

Assuming hierarchies of VEVs with $\mathrm{O}(1)$ coefficients,

$$
\begin{aligned}
\sigma_{4} & \ll 1, \\
-p \sigma_{3} & =f \frac{\sigma_{4} \sigma_{6}}{\sigma_{5}}+k \sigma_{3} \sigma_{21} \simeq O\left(10^{-3}\right), \\
c \sigma_{3}+e \sigma_{3} \sigma_{21} & \simeq O\left(10^{-3}\right), \\
a \sigma_{6} \sigma_{9} & \simeq O\left(10^{-5}\right), \\
\sigma_{21} & \simeq O\left(\frac{a}{b}\right) \ll 1, \\
r \sigma_{21} & \simeq O(1), \\
p \sim c \sim e \sim f \sim k \sim \ell \sim r & \simeq O(1),
\end{aligned}
$$

we estimate

$$
\approx\left(\begin{array}{ccc}
0, & p \sigma_{3}, & 0 \\
c \sigma_{3}, & f \frac{\sigma_{4} \sigma_{6}}{\sigma_{5}}, & \ell \sigma_{3} \sigma_{4} \\
a \sigma_{6} \sigma_{9}, & b \sigma_{6} \sigma_{9} \sigma_{21}, & 1
\end{array}\right)
$$

which can be close to Eq. (66). Let all singlet VEVs are real except $\sigma_{9}$ and $\sigma_{21}$ [23],

$$
\sigma_{9}=\left|\sigma_{9}\right| e^{i \theta} \simeq\left|\sigma_{9}\right| e^{\frac{i \pi}{2}}, \quad \sigma_{21}=\left|\sigma_{21}\right| e^{i \phi} .
$$

The phase of $e^{i(\theta+\phi)}$ is fitted to the phase of

$$
-1.15 \times 10^{-3}+\left(2.88 \times 10^{-4}-0.534 \times 10^{-4} t_{5}\right) e^{i \delta} \sim e^{i(\theta+\phi)}
$$

In Table $\mathrm{V}$, we list $\theta+\phi$ for a few $t_{5}$. For $\delta_{\mathrm{CKM}}=\frac{\pi}{2}$ and $t_{5} \simeq 5.5$, we obtain $\phi \simeq-\theta$. Irrespective of the value of $\phi$, the $C P$ phase in the Jarlskog determinant, $\delta_{\mathrm{CKM}}$, is the phase in $M_{u(31)}^{w}$ with the KS parametrization given in Eq. (22).

\section{E. $C P$ phases in the quark and lepton sectors}

As done before, let us diagonalize the symmetric fermion masses first. In the flipped SU(5) model, therefore, we diagonalize down-type quark masses and neutrino masses. Then, we consider up-type quarks and charged leptons. Then, the $(3,1)$ elements of the mass matrices are the key. For the third family members from $U$, masses of $t$ quark and $\tau$ lepton arise from

TABLE V. Phases of $M_{u(32)}^{w}$ for $\delta=\frac{\pi}{2}$.

\begin{tabular}{lcc}
\hline \hline$t_{5}$ & $\frac{\left(-0.288+0.0534 t_{5}\right)}{1.15}$ & $\theta+\phi$ \\
\hline 0 & -0.250 & $-14.04^{\circ},-0.244 \pi$ \\
5.5 & 0.0054 & $0.286^{\circ}, \sim 0$ \\
10 & 0.214 & $12.0^{\circ}, 0.209 \pi$ \\
\hline \hline
\end{tabular}




$$
\begin{array}{ll}
\overline{\mathbf{1 0}}_{-1}(U) \leftrightarrow \mathbf{5}_{+3}(U) \times \mathbf{5}_{-2}\left(T_{6}\right), & \left(t \text { quark: } \sum_{\text {Sect } T_{i}}=6, \sum Q_{\text {anom }}=+14\right) \\
\mathbf{5}_{+3}(U) \leftrightarrow \mathbf{1}_{-5}(U) \times \overline{\mathbf{5}}_{+2}\left(T_{6}\right), & \left(\tau \text { lepton: } \sum_{\text {Sect } T_{i}}=6, \sum Q_{\text {anom }}=-4\right)
\end{array}
$$

The phenomenologically determined leptonic mass element $M_{e(31)}^{w}$ can be obtained from Eq. (61) by changing the quark parameters $\theta_{i}, \delta, \Delta, m_{u}, m_{c}, m_{t}$ to leptonic parameters of Eq. (35): $\Theta_{i}, \delta_{L}, \Delta_{L}, m_{e}, m_{\mu}, m_{\tau}$. Choose the leptonic $V$ matrix elements such that

$$
\frac{S_{4}}{C_{4}}=\frac{S_{1}}{C_{1}}, \quad \frac{S_{5}}{C_{5}}=\frac{m_{\mu}}{m_{\tau}} \frac{S_{2}}{C_{2}}, \quad S_{6}=0, \quad \Delta_{L}=\delta_{L} .
$$

Then, $M_{e(31)}^{w} / m_{\tau} \simeq-\sin \Theta_{1} \sin \Theta_{2} e^{i \delta_{L}}$ where $\delta_{L}$ is the PMNS phase.

In our model, Table IV, there are three $e^{c}$ fields in the leptonic case (instead of four $u^{c}$ fields in the quark case), and we can choose $S_{24}^{A}$ which is the antisymmetric combination of $S_{24 a}$ and $S_{24 b}$ in $T_{4}^{0}$. So, the leptonic mass matrix has four zero entries with the antisymmetric 1 st row and antisymmetric 2nd column,

$$
\left(\begin{array}{ccc}
0, & M_{e(12)}^{w}, & 0 \\
M_{e(21)}^{w}, & 0, & M_{e(23)}^{w} \\
M_{e(31)}^{w}, & 0, & M_{e(33)}^{w}
\end{array}\right)
$$

For the lepton phase, we need $M_{e(31)}^{w}$ whose phase is $\delta_{L}{ }^{6}$

$$
\begin{aligned}
M_{e(31)}^{w}: & C_{1}\left(\mathbf{5}_{U}\right) S_{24}^{S}\left(\mathbf{1}_{T_{4}^{0}}\right) H_{d}\left(\overline{\mathbf{5}}_{T_{6}}\right) \\
& \left(\sum_{\text {Sect } T_{i}}=10, \sum Q_{\text {anom }}=-4\right)
\end{aligned}
$$

where $S_{24}^{S}$ is the symmetric combination of $S_{24 a}$ and $S_{24 b}$. So, the product of FN singlets must satisfy

$$
\sum_{\text {Sect } T_{i}}=2 \bmod .12, \quad \sum Q_{\text {anom }}=+4 .
$$

It is satisfied by $\sigma_{5} \sigma_{6} \sigma_{9}$ and $\sigma_{2} \sigma_{15} \sigma_{21}{ }^{7}$ In our vacuum, we choose $\left|\sigma_{21}\right|$ somewhat smaller than $\left|\sigma_{9}\right|$ but large enough to achieve a successful doublet-triplet splitting, viz. Eq. (45). Therefore, the phase $\delta_{L}$ of $M_{e(31)}^{w}$ is mostly given by the phase of $\sigma_{9}$ as in $M_{u(31)}^{w}$. In the leptonic case, this $\delta_{L}$ is $\delta_{\text {PMNS }}$. But, for this interpretation to work, $\sigma_{9}$ should not appear in the neutrino mass matrix such that $U_{\mathrm{PMNS}}=$ $U_{\nu}^{\dagger} U_{e}$ contains $\sigma_{9}$ only in $U_{e}$. The neutrino mass matrix is of the form $\mathbf{5}_{+3} M_{\nu}^{w} \mathbf{5}_{+3}$ which can arise from the following couplings,

$$
\begin{aligned}
& \frac{1}{M^{9}} C_{3}\left[\mathbf{5}_{+3}\left(T_{4}^{0}\right)\right] C_{3}\left[\mathbf{5}_{+3}\left(T_{4}^{0}\right)\right] H_{u}\left[\mathbf{5}_{-2}\left(T_{6}\right)\right] H_{u}\left[\mathbf{5}_{-2}\left(T_{6}\right)\right] C_{11}\left[\overline{\mathbf{1 0}}_{-1}\left(T_{3}\right)\right] C_{11}\left[\overline{\mathbf{1 0}}_{-1}\left(T_{3}\right)\right] \\
& \quad \cdot \sigma_{3}\left[\mathbf{1}\left(T_{4}^{0}\right)\right] \sigma_{3}\left[\mathbf{1}\left(T_{4}^{0}\right)\right] \sigma_{5}\left[\mathbf{1}\left(T_{6}\right)\right] \sigma_{5}\left[\mathbf{1}\left(T_{6}\right)\right] \sigma_{21}\left[\mathbf{1}\left(T_{1}^{0}\right)\right] \sigma_{21}\left[\mathbf{1}\left(T_{1}^{0}\right)\right] \\
& \frac{1}{M^{7}} C_{3}\left[\mathbf{5}_{+3}\left(T_{4}^{0}\right)\right] C_{1}\left[\mathbf{5}_{+3}(U)\right] H_{u}\left[\mathbf{5}_{-2}\left(T_{6}\right)\right] H_{u}\left[\mathbf{5}_{-2}\left(T_{6}\right)\right] C_{11}\left[\overline{\mathbf{1 0}}_{-1}\left(T_{3}\right)\right] C_{11}\left[\overline{\mathbf{1 0}}_{-1}\left(T_{3}\right)\right] \\
& \quad \cdot \sigma_{5}\left[\mathbf{1}\left(T_{6}\right)\right] \sigma_{6}\left[\mathbf{1}\left(T_{6}\right)\right] \sigma_{21}\left[\mathbf{1}\left(T_{1}^{0}\right)\right] \sigma_{21}\left[\mathbf{1}\left(T_{1}^{0}\right)\right] \\
& \frac{1}{M^{8}} C_{1}\left[\mathbf{5}_{+3}(U)\right] C_{1}\left[\mathbf{5}_{+3}(U)\right] H_{u}\left[\mathbf{5}_{-2}\left(T_{6}\right)\right] H_{u}\left[\mathbf{5}_{-2}\left(T_{6}\right)\right] C_{11}\left[\overline{\mathbf{1 0}}_{-1}\left(T_{3}\right)\right] C_{11}\left[\overline{\mathbf{1 0}}_{-1}\left(T_{3}\right)\right] \\
& \quad \cdot \sigma_{2}\left[\mathbf{1}\left(T_{4}^{0}\right)\right] \sigma_{5}\left[\mathbf{1}\left(T_{6}\right)\right] \sigma_{6}\left[\mathbf{1}\left(T_{6}\right)\right] \sigma_{21}\left[\mathbf{1}\left(T_{1}^{0}\right)\right] \sigma_{21}\left[\mathbf{1}\left(T_{1}^{0}\right)\right]
\end{aligned}
$$

\footnotetext{
${ }^{6}$ We have already presented $M_{u(31)}^{w}$ with phase $\delta_{\mathrm{CKM}}$. We have this definite statement because we used the KS parametrization, Eqs. (22) and (35), of mixing angles [23].

${ }^{7}$ In $M_{u(31)}^{w}$, we did not include $\sigma_{10}$ in addition to $\sigma_{9}$ for simplicity. Namely, it is equivalent to assuming $\left\langle\sigma_{10}\right\rangle=0$ or $\sigma_{10}=\left|\sigma_{10}\right| e^{i \theta}$. In $M_{e(31)}^{w}$ also, we consider only $\sigma_{9}$ for simplicity.
}

where $\sigma_{9}$ does not appear. So, the phase in $\sigma_{9}$ is the PMNS phase. The generic magnitudes of masses from the above couplings are $\left(v_{\mathrm{ew}}^{2} / M\right)(V / M)^{8,6,7}$ where $V$ and $M$ are some scales around/above the GUT scale, and we can obtain reasonable strength for neutrino masses.

Equation (77) shows that the L-handed up-type quarks, appearing in $\overline{\mathbf{1 0}}_{-1}$, use charge lowering operators to couple to $W_{\mu}^{-}$and the L-handed charged leptons, appearing in $\mathbf{5}_{+3}$, 
use charge raising operators to couple to $W_{\mu}^{+}$. So, we must consider the same charge charged-gauge boson $W_{\mu}$ to compare the signs of $\delta_{\mathrm{CKM}}$ and $\delta_{\mathrm{PMNS}}$. Also, we must specify the signs of the effective Yukawa couplings in $M_{u(31)}^{w}$ and $M_{e(31)}^{w}$ dictated by string compactification. At this stage, we allow any sign for $M_{u(31)}^{w}$ and $M_{e(31)}^{w}$ since we considered only the selection rules. If the signs of $M_{u(31)}^{w}$ and $M_{e(31)}^{w}$ are the same (opposite), then we conclude that $\delta_{\mathrm{CKM}}$ and $\delta_{\mathrm{PMNS}}$ have the opposite (same) signs. ${ }^{8}$ The case of opposite signs is consistent with the currently favored phases of $\delta_{\mathrm{CKM}}$ [23] and $\delta_{\text {PMNS }}$ [24].

In the PS type standard model $\mathrm{SU}(4) \times \mathrm{SU}(2)_{L} \times$ $\mathrm{SU}(2)_{R}$, we would have fermion matter spectra, containing quark and lepton doublets,

$$
(\mathbf{4}, \mathbf{2}, \mathbf{1})_{L} \oplus(\mathbf{4}, \mathbf{1}, \mathbf{2})_{R}+\cdots
$$

Suppose that the Yukawa coupling $(\mathbf{4}, \mathbf{2}, \mathbf{1})_{L} \times\left(\mathbf{4}^{*}, \mathbf{1}, \mathbf{2}\right)_{L} \times$ $(\mathbf{1}, \mathbf{2}, \mathbf{2})_{h}$ via Higgs $(\mathbf{1}, \mathbf{2}, \mathbf{2})_{h}$ is present from the orbifold compactification. Then, the Yukawa coupling arises from the L-handed Higgs field doublets $\epsilon^{i j}(\mathbf{1}, \mathbf{2},(i j))_{h}=$ $(\mathbf{1}, \mathbf{2},(12))_{h}-(\mathbf{1}, \mathbf{2},(21))_{h}$ where the R-hand index (12) gives the Higgs doublet coupling to quark doublets and the R-hand index (21) gives the Higgs doublet coupling to lepton doublets. We use the same charge $\mathrm{W}$, i.e., $W_{\mu}^{+}$, for coupling to down-type quarks and charged leptons. So, the relative signs of $M_{d(31)}^{w}$ and $M_{e(31)}^{w}$ are opposite if the product with FN singlet contributions give the same sign. If we use the mass matrices of $M_{d(31)}^{w}$ and $M_{e(31)}^{w}$ for asymmetric mass matrices as in the GG model, then $\delta_{\mathrm{CKM}}$ and $\delta_{\text {PMNS }}$ have the opposite signs. But, here one needs an example for breaking $\mathrm{SO}(10)$ down to $\mathrm{SU}(4) \times \mathrm{SU}(2)_{L} \times$ $\mathrm{SU}(2)_{R}$, where the rank is not reduced, from the spectra of orbifold compactification. One may use the bulk fields for an adjoint representation as pointed out for $\mathbf{Z}_{6-I I}$ in

\footnotetext{
${ }^{8}$ In the GG model [37], the symmetric quark mass matrices are for neutrinos and up-type quarks. The asymmetric quark mass matrices are for the down-type quarks and charged leptons via the same coupling $\overline{\mathbf{1 0}}_{0} \mathbf{5}_{0} H_{0 d}$, and if we had tried the strategy we chose here then we would have obtained the same sign for $\delta_{\mathrm{CKM}}$ and $\delta_{\text {PMNS }}$ irrespective of the signs of $M_{u(31)}^{w}$ and $M_{e(31)}^{w}$. But this idea is not workable in the GG model because we lack an adjoint representation for breaking $\mathrm{SU}(5)$ down to the SM.
}

Ref. [45] and for $\mathbf{Z}_{2} \times \mathbf{Z}_{2}$ in Ref. [52] where the $N=2$ gauge multiplet in an effective 5-dimensional SUSY model allows an adjoint representation of spin- 0 fields.

\section{CONCLUSION}

In this paper, we presented a theory toward understanding the quark and lepton mixing angles. Specifically, we presented a working example obtained from a string compactification [47] with $Q_{\text {anom }}$ charge presented in [2]. Explicit presentations were given for the CKM matrix. The $(3,3)$ element of quark mass matrix in the weak basis, is assumed to be close to the $t$-quark mass. Because there are only three L-handed quark doublets in the model, the uptype quark mass matrix is antisymmetric under the exchange of $a \leftrightarrow b$ among R-handed flavor indices (or $u^{c}$ fields) obtained from $T_{4}^{0}$. This is because the multiplicity 2 for $\mathbf{5}_{-3}$ from $T_{4}^{0}$ is generic and there is no way to distinguish these two. The antisymmetric combination of $a$ and $b$ is named for the 1 st family member of $\mathbf{5}_{+3}$ 's. But, there are four L-handed up-type quark doublets and the uptype quark families have a freedom to choose from these four. We used the freedom of choosing the unitary matrix for the R-handed quarks to fit to the data, and showed that this model predicts reasonable mixing angles within experimental error bounds. Also, we studied the relation between $\delta_{\mathrm{CKM}}$ and $\delta_{\mathrm{PMNS}}$ by the phases of some SM singlet scalar fields, assuming that all Yukawa coupling constants from string compactification are real. For the proton decay problem, a $\mathbf{Z}_{2}$ matter parity cannot be introduced consistently with the solution of the doublet-triplet splitting problem by the GUT scale VEVs, $\left\langle\overline{\mathbf{1 0}}_{-1}\left(T_{3}\right)\right\rangle$ and $\left\langle\mathbf{1 0}_{+1}\left(T_{9}\right)\right\rangle$. But, we showed that the proton decay operator appears at a dimension 10 level, which can be made small enough while achieving the doublet-triplet splitting. It will be interesting if a kind of $\mathrm{R}$ parity is found within the scheme, which will be published soon [53].

\section{ACKNOWLEDGMENTS}

I have benefitted from discussions with S. Kim, B. Kyae, and S. Nam. This work is supported in part by the National Research Foundation (NRF) Grant No. NRF2015R1D1A1A01058449 and by the Institute of Basic Science (IBS-R017-D1-2014-a00).
[1] J. E. Kim, Towards unity of families: Anti-SU(7) from $\mathbf{Z}_{12-I}$ orbifold compactification, J. High Energy Phys. 06 (2015) 114.
[2] J. E. Kim, B. Kyae, and S. Nam, The anomalous U(1) anom symmetry and flavors from an $\mathrm{SU}(5) \times \mathrm{SU}(5)^{\prime} \mathrm{GUT}$ in $\mathbf{Z}_{12-I}$ orbifold compactification, Eur. Phys. J. C 77, 847 (2017). 
[3] J. E. Kim, A Model of Flavor Unity, Phys. Rev. Lett. 45, 1916 (1980); Flavor unity in SU(7): Low mass magnetic monopole, doubly charged lepton, and $\mathrm{Q}=5 / 3,4 / 3$ quarks, Phys. Rev. D 23, 2706 (1981).

[4] S. F. King and G. G. Ross, Fermion masses and mixing angles from SU(3) family symmetry, Phys. Lett. B 520, 243 (2001).

[5] D. B. Reiss, Can the family group be a global symmetry?, Phys. Lett. 115B, 217 (1982).

[6] F. Wilczek, Axions and Family Symmetry Breaking, Phys. Rev. Lett. 49, 1549 (1982).

[7] G. B. Gelmini, S. Nussinov, and T. Yanagida, Does nature like Nambu-Goldstone bosons?, Nucl. Phys. B219, 31 (1983).

[8] J. E. Kim, S. Nam, and Y. K. Semetzidis, Fate of global symmetries in the Universe: QCD axion, quintessential axion and trans-Planckian inflaton decay-constant, Int. J. Mod. Phys. A 33, 1830002 (2018).

[9] For an early review, see, P. Ramond, Fermion masses and multiplicity, Comments Nucl. Part. Phys. 16, 1 (1986).

[10] N. Cabibbo, Unitary Symmetry and Leptonic Decays, Phys. Rev. Lett. 10, 531 (1963).

[11] M. Kobayashi and T. Maskawa, $C P$ violation in the renormalizable theory of weak interaction, Prog. Theor. Phys. 49, 652 (1973).

[12] B. Pontecorvo, Zh. Eksp. Teor. Fiz. 34, 247 (1957) [Inverse beta processes and nonconservation of lepton charge, Sov. Phys. JETP 7, 172 (1957)].

[13] Z. Maki, M. Nakagawa, and S. Sakata, Remarks on the unified model of elementary particles, Prog. Theor. Phys. 28, 870 (1962).

[14] S. Weinberg, The problem of mass, Trans. N.Y. Acad. Sci. 38, 185 (1977).

[15] H. Fritzsch, Weak interaction mixing in the six-quark theory, Phys. Lett. 73B, 317 (1978).

[16] F. Wilczek and A. Zee, Discrete flavor symmetries and a formula for the Cabibbo angle, Phys. Lett. 70B, 418 (1977); Erratum, Phys. Lett. 72B, 504(E) (1978).

[17] C. D. Froggatt and H. B. Nielsen, Hierarchy of quark masses, Cabibbo angles and $C P$ violation, Nucl. Phys. B147, 277 (1979).

[18] H. Georgi, Towards a grand unified theory of flavor, Nucl. Phys. B156, 126 (1979).

[19] P. Frampton, SU(N) grand unification with several quark lepton generations, Phys. Lett. 88B, 299 (1979).

[20] P. Frampton and S. Nandi, SU(9) Grand Unification of Flavor with Three Generations, Phys. Rev. Lett. 43, 1460 (1979).

[21] P. F. Harrison, D. H. Perkins, and W. G. Scott, Threefold maximal lepton mixing and the solar and atmospheric neutrino deficits, Phys. Lett. B 349, 137 (1995).

[22] E. Ma and G. Rajasekaran, Softly broken $A_{4}$ symmetry for nearly degenerate neutrino masses, Phys. Rev. D 64, 113012 (2001).

[23] J. E. Kim and M.-S. Seo, Parametrization of the CKM matrix, Phys. Rev. D 84, 037303 (2011); J. E. Kim, D. Y. Mo, and S. Nam, Final state interaction phases obtained by data from $C P$ asymmetries, J. Korean Phys. Soc. 66, 894 (2015).
[24] K. Abe et al. (T2K Collaboration), Combined Analysis of Neutrino and Antineutrino Oscillations at T2K, Phys. Rev. Lett. 118, 151801 (2017).

[25] J. E. Kim and S. Nam, Unifying $C P$ violations of quark and lepton sectors, Eur. Phys. J. C 75, 619 (2015).

[26] C. D. Froggatt and H. B. Nielsen, Hierarchy of quark masses, Cabibbo angles and $C P$ violation, Nucl. Phys. B147, 277 (1979).

[27] D. J. Gross, J. A. Harvey, E. J. Martinec, and R. Rohm, The Heterotic String, Phys. Rev. Lett. 54, 502 (1985).

[28] K.-S. Choi and J.E. Kim, Quarks and Leptons from Orbifolded Superstring, Lecture Notes in Physics (Springer-Verlag, New York, 2006), Vol. 696, Chap. 10 and Appendix D.

[29] J. E. Kim and B. Kyae, Flipped SU(5) from $\mathbf{Z}_{12-I}$ orbifold with Wilson line, Nucl. Phys. B770, 47 (2007).

[30] L. J. Dixon, V. Kaplunovsky, and J. Louise, On effective field theories describing $(2,2)$ vacua of the heterotic string, Nucl. Phys. B329, 27 (1990).

[31] F. Gmeiner, R. Blumenhagen, G. Honecker, D. Lust, and T. Weigand, One in a billion: MSSM-like D-brane statistics, J. High Energy Phys. 01 (2006) 004.

[32] M. R. Douglas and W. Taylor, The landscape of intersecting brane models, J. High Energy Phys. 01 (2007) 031.

[33] O. Lebedev, H. P. Nilles, S. Raby, S. Ramos-Sanchez, M. Ratz, P. K. S. Vaudrevange, and A. Wingerter, Low Energy Supersymmetry from the Heterotic Landscape, Phys. Rev. Lett. 98, 181602 (2007).

[34] O. Lebedev, H. P. Nilles, S. Ramos-Sanchez, M. Ratz, and P. K. S. Vaudrevange, Heterotic mini-landscape (II): Completing the search for MSSM vacua in a $\mathbf{Z}_{6}$ orbifold, Phys. Lett. B 668, 331 (2008).

[35] J.E. Kim, Light pseudoscalars, particle physics and cosmology, Phys. Rep. 150, 1 (1987).

[36] H. Georgi, H. R. Quinn, and S. Weinberg, Hierarchy of Interactions in Unified Gauge Theories, Phys. Rev. Lett. 33, 451 (1974).

[37] H. Georgi and S. L. Glashow, Unity of All Elementary Particle Forces, Phys. Rev. Lett. 32, 438 (1974).

[38] A. J. Buras, J. Ellis, M. K. Gaillard, and D. V. Nanopoulos, Aspects of the grand unification of strong, weak and electromagnetic interactions, Nucl. Phys. B135, 66 (1978).

[39] H. Georgi and C. Jarlskog, A new lepton-quark mass relation in a unified theory, Phys. Lett. 86B, 297 (1979).

[40] S. M. Barr, A new symmetry breaking pattern for $\mathrm{SO}(10)$ and proton decay, Phys. Lett. 112B, 219 (1982).

[41] J. P. Derendinger, J.E. Kim, and D. V. Nanopoulos, Anti-SU(5), Phys. Lett. 139B, 170 (1984).

[42] H. Georgi, The state of the art-Gauge theories, AIP Conf. Proc. 23, 575 (1975).

[43] H. Fritszch and P. Minkowski, Unified interactions of leptons and hadrons, Ann. Phys. (N.Y.) 93, 193 (1975).

[44] J. C. Pati and Abdus Salam, Unified lepton-hadron symmetry and a gauge theory of the basic interactions, Phys. Rev. D 8, 1240 (1973).

[45] T. Kobayashi, S. Raby, and R.-J. Zhang, Searching for realistic $4 d$ string models with a Pati-Salam symmetry: Orbifold grand unified theories from heterotic string compactification on a $\mathbf{Z}_{6}$ orbifold, Nucl. Phys. B704, 3 (2005). 
[46] I. Antoniadis, J. R. Ellis, J . S. Hagelin, and D. V. Nanopoulos, The flipped $\mathrm{SU}(5) \times \mathrm{U}(1)$ string model revamped, Phys. Lett. B 231, 65 (1989).

[47] J. H. Huh, J. E. Kim, and B. Kyae, SU(5) flip x SU(5)' from $\mathbf{Z}_{12-I}$, Phys. Rev. D 80, 115012 (2009).

[48] C. Jarlskog, Commutator of the Quark Mass Matrices in the Standard Electroweak Model and a Measure of Maximal CP Nonconservation, Phys. Rev. Lett. 55, 1039 (1985).

[49] H. M. Lee, S. Raby, M. Ratz, G. R. Ross, R. Schieren, K. Schmidt-Hoberg, and P. K. S. Vaudrevange, Discrete R symmetries for the MSSM and its singlet extensions, Nucl. Phys. B850, 1 (2011); M. Paraskevas and K. Tamvakis, On discrete R-symmetries in MSSM and its extensions,
Phys. Rev. D 86, 015009 (2012); J. E. Kim, Abelian discrete symmetries $\mathbf{Z}_{N}$ and $\mathbf{Z}_{n R}$ from string orbifolds, Phys. Lett. B 726, 450 (2013).

[50] R. Dermisek, A. Mafi, and S. Raby, SUSY GUTs under siege: Proton decay, Phys. Rev. D 63, 035001 (2000).

[51] S. Raby, Supersymmetric Grand Unified Theories: From Quarks to Strings via SUSY GUTs, Lecture Notes in Physics (Springer-Verlag, New York, 2017), Vol. 939.

[52] A. E. Faraggi, C. Kounas, and J. Rizos, Chiral family classification of fermionic $\mathbf{Z}_{2} \times \mathbf{Z}_{2}$ heterotic orbifold models, Phys. Lett. B 648, 84 (2007).

[53] J.E. Kim, R parity from string compactification (to be published). 